\title{
Higgs diphoton rate enhancement from supersymmetric physics beyond the MSSM
}

\author{
Marcus Berg $^{a}$, Igor Buchberger ${ }^{a}$, D. M. Ghilencea ${ }^{b, c}$, Christoffer Petersson $^{d, e}$ \\ ${ }^{a}$ Department of Physics, Karlstad University, 65188 Karlstad, Sweden. \\ ${ }^{b}$ Theory Division, CERN, 1211 Geneva 23, Switzerland. \\ ${ }^{c}$ Theoretical Physics Department, National Institute of Physics \\ and Nuclear Engineering (IFIN-HH) Bucharest MG-6, 077125 Romania. \\ ${ }^{d}$ Physique Théorique et Mathématique, Université Libre de Bruxelles, \\ C.P. 231, 1050 Bruxelles, Belgium. \\ ${ }^{e}$ International Solvay Institutes, Brussels, Belgium.
}

\begin{abstract}
We show that supersymmetric "new physics" beyond the MSSM can naturally accommodate a Higgs mass near $126 \mathrm{GeV}$ and enhance the signal rate in the $h \rightarrow \gamma \gamma$ channel, while the signal rates in all the other Higgs decay channels coincide with Standard Model expectations, except possibly the $h \rightarrow Z \gamma$ channel. The "new physics" that corrects the relevant Higgs couplings can be captured by two supersymmetric effective operators. We provide a simple example of an underlying model in which these operators are simultaneously generated. The scale of "new physics" that generates these operators can be around $5 \mathrm{TeV}$ or larger, and outside the reach of the LHC.
\end{abstract}

marcus.berg@kau.se, igorbuch@kau.se, dumitru.ghilencea@cern.ch, christoffer.petersson@ulb.ac.be 


\section{Introduction}

The ATLAS and CMS collaborations at CERN recently presented strong experimental evidence for a Higgs-like resonance around $126 \mathrm{GeV}$ [1], marking a historic achievement in particle physics. The signal rates in the $Z Z^{*}$ and $W W^{*}$ channels are in good agreement with the Standard Model (SM) predictions. The $b \bar{b}$ and $\tau^{+} \tau^{-}$signal rates are also compatible with the SM, although with substantial error bars. At the time of writing, the signal rate in the $h \rightarrow \gamma \gamma$ channel is about 1.5 - 2 times larger than the SM prediction. This discrepancy is only at the level of two standard deviations and there are theoretical uncertainties [2]. Nevertheless, it is interesting to contemplate whether physics beyond the SM (BSM) can be responsible for this discrepancy (for recent work in this direction, see [3]).

In this work we shall assume that the excess in the diphoton channel is due to BSM physics that has negligible effect on channels other than $h \rightarrow \gamma \gamma$. Indeed, this channel is sensitive to new physics, since it is a loop-level process in the SM. With this in mind, we shall focus on the minimal supersymmetric (SUSY) extension of the SM (MSSM) close to the "decoupling limit" [4, in which the lightest neutral CP-even Higgs boson $h$ is SM-like.

It is surprising that, despite its large number of parameters, the MSSM has difficulties in accommodating an enhancement of the $h \rightarrow \gamma \gamma$ partial decay width $\Gamma_{h \gamma \gamma}$ without affecting the other partial decay widths. In fact this requirement seems to single out loop-induced contributions from very light color singlet superpartners with a significant coupling to the Higgs, meaning strongly mixed light stau sleptons, at around $100 \mathrm{GeV}$ [5]. However, this introduces issues with vacuum stability and may even be possible to rule out at the LHC. In addition, large radiative corrections are needed to obtain a mass of $m_{h} \approx 126 \mathrm{GeV}$ for the lightest Higgs of the MSSM. This requires large supersymmetry breaking terms, such as $\mathrm{TeV}$ stop masses and/or a large top $A$-term. The lack of evidence for superpartners in the direct SUSY searchers at the LHC also indicates that soft terms should be large. However, large supersymmetry breaking terms lead to severe fine-tuning [6, 7] in most versions of the $\operatorname{MSSM}^{1}$ (with or without universal gaugino masses, or Higgs soft masses different from $m_{0}$ and also from each other). This situation suggests that a solution to this problem is not in the SUSY breaking sector but rather in the one that preserves supersymmetry. Following this idea, the purpose of this work is to answer whether one can have a minimal supersymmetric extension of the Higgs sector that allows $m_{h} \approx 126 \mathrm{GeV}$ without undue fine tuning and a simultaneous $h \rightarrow \gamma \gamma$ enhancement, while complying with the negative SUSY searches so far.

To this end, one way to proceed is suggested by minimal extensions of the MSSM Higgs sector, like the Next-to-MSSM (NMSSM) model which contains an additional singlet chiral superfield (see [9] for a review), and by models where the soft $B \mu$ term is promoted to a SUSY operator [10]. In the NMSSM an enhancement of the $h \rightarrow \gamma \gamma$ branching ratio is possible (although this also alters other couplings) beyond the SM level [11. However, the NMSSM remains badly fine-tuned (fine tuning $\Delta>200$ ) for $m_{h} \approx 126 \mathrm{GeV}$ [12]. There are

\footnotetext{
${ }^{1}$ For a discussion of the negative impact of the EW fine tuning on the $\chi^{2}$ fit of such models see also 8 .
} 
known ways to bypass this problem such as in the so-called "generalized" version of NMSSM (GNMSSM) with a superpotential mass term for the singlet superfield, where the electroweak fine tuning is significantly reduced to more acceptable levels $(\Delta \approx 30)[12,13$, 14. These are examples of explicit supersymmetry-preserving modifications of the MSSM that can render more natural the interpretation of the resonance at $126 \mathrm{GeV}$ as the lightest Higgs.

In this work, instead of considering such specific models, we shall relax the rigid, minimal structure of the MSSM Higgs sector and perform an effective field theory analysis of the most relevant SUSY-preserving operators in this sector. This approach should recover, in a particular region of the parameter space, scenarios such as those presented above (GNMSSM, etc). We show that it is possible to naturally accommodate a Higgs mass of $126 \mathrm{GeV}$, an enhanced Higgs coupling to photons, and simultaneously SM-like Higgs couplings to the other particles, using only a few supersymmetry-preserving operators with small coefficients.

In general there is a large set of operators that one could consider in the Higgs sector [15. Regarding the Higgs mass it is known that the presence of the effective dimension 5 superpotential operator [16, 17]

$$
\frac{1}{M}\left(H_{u} \cdot H_{d}\right)^{2}
$$

can accommodate $m_{h} \approx 126 \mathrm{GeV}$ without undue fine tuning [13]. The suppression scale $M$ represents the mass scale of the SUSY degrees of freedom that have been integrated out to generate (1.1). Concerning $\Gamma_{h \gamma \gamma}$, from the list of effective operators of dimensions $d=5$ and $d=6$ in the Higgs sector [13, 18, one notices the presence of a SUSY effective operator

$$
\frac{1}{M^{2}}\left(H_{u} \cdot H_{d}\right) \operatorname{Tr}\left(W^{\alpha} W_{\alpha}\right)
$$

that can significantly modify the $h \rightarrow \gamma \gamma$ rate. Here $W_{\alpha}$ is the electroweak gauge field strength superfield. Based on these observations we intend to investigate closer the phenomenological impact of (1.1) and (1.2). As a simple example will show, these operators can be generated simultaneously by an underlying microscopic model, making their combination rather natural. The effect of both operators is maximized for small $\tan \beta$, where the MSSM tree level contribution to the Higgs mass is minimized. This means that the impact of the $d=5$ operator in accommodating an $m_{h} \approx 126 \mathrm{GeV}$ is rather significant. To our knowledge, the particular combination of the effective SUSY operators in $(1.1)$ and $(1.2)$ has not been studied in the past for the problems we address ${ }^{2}$

The paper is organized as follows. In Section 2 we calculate the corrections from the effective operators to the Higgs mass and mixing angle. In Section 3 we discuss how these operators correct the Higgs couplings and signal rates, with focus on the decoupling limit. The results in terms of the Higgs mass and the partial widths for the $h \rightarrow \gamma \gamma$ and $h \rightarrow Z \gamma$ channels are discussed in Section 4 . Section 5 provides an example of the origin of the effective

\footnotetext{
${ }^{2}$ The operator in 1.2 was separately discussed in [19].
} 
operators and Section 6 contains our conclusions, while some details concerning the on-shell Lagrangian are given in the Appendix.

\section{Corrections from SUSY operators to the Higgs couplings}

The effective model we consider consists of the usual MSSM Higgs sector, extended by the operators discussed in the introduction. The relevant part of the Lagrangian is, in standard notation,

$$
\mathcal{L}=\int d^{4} \theta \sum_{i=u, d}\left(1-m_{i}^{2} \theta^{2} \bar{\theta}^{2}\right) H_{i}^{\dagger} e^{V_{i}} H_{i}+\left(\int d^{2} \theta \mu\left(1+B \theta^{2}\right) H_{d} \cdot H_{u}+\text { h.c. }\right)+\mathcal{O}_{5}+\mathcal{O}_{6}
$$

where the chiral superfields have components $H_{i} \equiv\left(h_{i}, \psi_{i}, F_{i}\right)$, and $m_{i}$ and $B$ are the soft terms. $\mathcal{O}_{5}$ is the only operator of dimension 5 that one can write in the Higgs sector, up to non-linear field redefinitions [20], and has the form:

$$
\mathcal{O}_{5}=\frac{c_{0}}{M} \int d^{2} \theta\left(H_{u} \cdot H_{d}\right)^{2}+\text { h.c. }
$$

For the component fields expression of $\mathcal{O}_{5}$ see eq. A.3.

There is a long list of operators in the Higgs sector of dimension $d=6[13,18,20$. A careful analysis of these operators shows that of all these there is one of them that can couple, in a supersymmetric way, to two gauge bosons:

$$
\mathcal{O}_{6}=\frac{1}{M^{2}} \sum_{s=1,2} \frac{c_{s}}{16 g_{s}^{2} \kappa_{s}} \int d^{2} \theta \operatorname{Tr}\left(W^{\alpha} W_{\alpha}\right)_{s}\left(H_{u} \cdot H_{d}\right)+\text { h.c. }
$$

Here $g_{1}$ and $g_{2}$ denote the $\mathrm{U}(1)_{Y}$ and $\mathrm{SU}(2)_{L}$ gauge couplings, respectively, and $\kappa_{s}$ is a constant that cancels the trace factor. $W^{\alpha}$ is the SUSY field strength of the $\mathrm{U}(1)_{Y}\left(\mathrm{SU}(2)_{L}\right)$ vector superfield $V_{1}\left(V_{2}\right)$ of components $\left(\lambda_{s}, V_{s, \mu}, D_{s} / 2\right), s=1,2 . \quad \mathcal{O}_{5}$ and $\mathcal{O}_{6}$ provide a minimal set of operators that is enough for our purposes. One can also consider SUSY breaking effects associated to these operators (see the appendix, eq. A.3), but we only seek supersymmetric solutions to our problem. The effective expansion is reliable when $c_{0,1,2}=\mathcal{O}(1)$ and $M$ is the largest scale in the theory. One can choose one of $c_{0,1,2}$, for example $c_{0}$, and set it to $c_{0}=1$ by redefining $M$. But it is useful to keep $c_{0}$ to easily trace or turn off the effects of $\mathcal{O}_{5}$. Also, to modify the diphoton rate $c_{1}$ or $c_{2}$ (or a combination thereof) is enough, so together with the scale $M$ we effectively have only two parameters. Keeping both $c_{1,2}$ generates an additional interesting coupling, see later.

Additional operators of $d=6$ can be present. Although they could have an impact on the Higgs mass [18, they have an additional scale suppression relative $\operatorname{td}^{3} \mathcal{O}_{5}$. There is an

\footnotetext{
${ }^{3}$ Strictly speaking, this is true for small $\tan \beta$ region, that will actually be the relevant region in our case.
} 
operator similar to $O_{6}$ but involving instead the $\mathrm{SU}(3)_{C}$ gauge group, that we do not consider here; this would change dramatically the Higgs decay rate to gluons, away from the SM values. We take the good agreement with the SM in most channels as evidence that if present, the coefficient of this operator must be small. Finally, another reason to restrict our analysis to $\mathcal{O}_{5,6}$ is that, as discussed later, they can be simultaneously generated by underlying physics.

\subsection{The on-shell Lagrangian}

The calculation of the on-shell Higgs Lagrangian extended by $\mathcal{O}_{5}$ and $\mathcal{O}_{6}$ is detailed in the appendix. The result is

$$
\begin{aligned}
\mathcal{L} & =-\frac{1}{2}\left[D_{2}^{a} D_{2}^{a}\left(1+\frac{c_{2}}{2 M^{2}}\left(h_{u} \cdot h_{d}+\text { h.c. }\right)\right)+(2 \rightarrow 1)\right] \\
& -\left|\mu+2 \frac{c_{0}}{M} h_{d} \cdot h_{u}\right|^{2}\left(\left|h_{d}\right|^{2}+\left|h_{u}\right|^{2}\right)+\left[\frac{\mu}{4}\left(\frac{c_{2}}{M^{2}} \lambda_{2}^{a} \lambda_{2}^{a}+\frac{c_{1}}{M^{2}} \lambda_{1}^{2}\right)\left(\left|h_{d}\right|^{2}+\left|h_{u}\right|^{2}\right)+\text { h.c. }\right] \\
& +\left\{\frac{c_{2}}{4 M^{2}}\left(h_{u} \cdot h_{d}\right)\left[i\left(\lambda_{2}^{a} \sigma^{\mu} \mathcal{D}_{\mu} \bar{\lambda}_{2}^{a}-\mathcal{D}_{\mu} \bar{\lambda}_{2}^{a} \bar{\sigma}^{\mu} \lambda_{2}^{a}\right)\right]+\text { h.c. }+(2 \rightarrow 1)\right\} \\
& +\frac{c_{0}}{M}\left[2\left(h_{u} \cdot h_{d}\right)\left(\psi_{d} \cdot \psi_{u}\right)-\left(h_{u} \cdot \psi_{d}+\psi_{u} \cdot h_{d}\right)^{2}\right]+\text { h.c. } \\
& +\left\{\frac { c _ { 2 } } { 4 M ^ { 2 } } \left[-\frac{1}{2}\left(h_{u} \cdot h_{d}\right)\left(F_{2}^{a \mu \nu} F_{2 \mu \nu}^{a}+\frac{i}{2} \epsilon^{\mu \nu \rho \sigma} F_{2 \mu \nu}^{a} F_{2 \rho \sigma}^{a}\right)\right.\right. \\
& \left.\left.-\sqrt{2}\left(h_{u} \cdot \psi_{d}+\psi_{u} \cdot h_{d}\right) \sigma^{\mu \nu} \lambda_{2}^{a} F_{2 \mu \nu}^{a}-\psi_{u} \cdot \psi_{d} \lambda_{2}^{a} \lambda_{2}^{a}\right]+(2 \rightarrow 1)+\text { h.c. }\right\} \\
& +\left[\mu B\left(h_{d} \cdot h_{u}\right)+\text { h.c. }\right]-\tilde{m}_{d}^{2}\left|h_{d}\right|^{2}-\tilde{m}_{u}^{2}\left|h_{u}\right|^{2}
\end{aligned}
$$

where $\tilde{m}_{i}^{2}=m_{i}^{2}+|\mu|^{2}, i=u, d$. For the explicit form of $D_{2}^{a} D_{2}^{a}$ and $D_{1}^{2}$, see eqs. A.7, A.8).

Eq. 2.4 contains all the information one needs to extract the corrections to the Higgs masses and couplings. In particular, notice the presence of new, supersymmetric couplings:

$$
-\frac{1}{8}\left(h_{u} \cdot h_{d}\right)\left(\frac{c_{2}}{M^{2}} \operatorname{Tr} F_{2}^{2}+\frac{c_{1}}{M^{2}} \operatorname{Tr} F_{1}^{2}\right)-\left|\mu+2 \frac{c_{0}}{M} h_{d} \cdot h_{u}\right|^{2}\left(\left|h_{d}\right|^{2}+\left|h_{u}\right|^{2}\right)+\text { h.c. }
$$

which are important below. There are also direct Higgs-higgsino and higgsino-gaugino couplings that can be relevant for dark matter models. From (2.4) we find the Higgs scalar potential $V_{h}$

$$
\begin{aligned}
V_{h} & =\tilde{m}_{d}^{2}\left|h_{d}\right|^{2}+\tilde{m}_{u}^{2}\left|h_{u}\right|^{2}-\left[\mu B h_{d} \cdot h_{u}+\text { h.c. }\right]+\frac{g_{2}^{2}}{2}\left|h_{d}^{\dagger} h_{u}\right|^{2}\left[1+\frac{c_{2}}{2 M^{2}}\left(h_{d} \cdot h_{u}+\text { h.c. }\right)\right] \\
& +\frac{1}{8}\left(\left|h_{d}\right|^{2}-\left|h_{u}\right|^{2}\right)^{2}\left[g^{2}+\left[\left(h_{d} \cdot h_{u}\right)\left(\frac{g_{1}^{2} c_{1}}{M^{2}}+\frac{g_{2}^{2} c_{2}}{M^{2}}\right)+\text { h.c. }\right]\right]+4\left|\frac{c_{0}}{M}\right|^{2}\left|h_{d} \cdot h_{u}\right|^{2}\left(\left|h_{d}\right|^{2}+\left|h_{u}\right|^{2}\right) \\
& +\left[\left(2 \frac{c_{0}}{M} \mu^{*}\right)\left(\left|h_{d}\right|^{2}+\left|h_{u}\right|^{2}\right)\left(h_{d} \cdot h_{u}\right)+\text { h.c. }\right],
\end{aligned}
$$

which depends on two parameters: $c_{0}$ from the effective dimension 5 operator and the combination $\left(g_{1}^{2} c_{1}+g_{2}^{2} c_{2}\right)$ from the effective dimension 6 operator. Note that last term in the first line above does not contribute to the neutral Higgs sector masses. 
We also include dominant loop corrections, although they do not play the same crucial role they do in the MSSM. In the small $\tan \beta$ regime and for dominant top Yukawa coupling, the one-loop and leading two-loop correction to $V_{h}$ is [21,

$$
\Delta V_{h}=\frac{g^{2}}{8} \delta\left|h_{u}\right|^{4}
$$

where

$$
\begin{aligned}
\delta & \equiv \frac{3 h_{t}^{4}}{g^{2} \pi^{2}}\left[\ln \frac{M_{\tilde{t}}}{m_{t}}+\frac{X_{t}}{4}+\frac{1}{32 \pi^{2}}\left(3 h_{t}^{2}-16 g_{3}^{2}\right)\left(X_{t}+2 \ln \frac{M_{\tilde{t}}}{m_{t}}\right) \ln \frac{M_{\tilde{t}}}{m_{t}}\right] \\
X_{t} & \equiv \frac{2\left(A_{t}-\mu \cot \beta\right)^{2}}{M_{\tilde{t}}^{2}}\left(1-\frac{\left(A_{t}-\mu \cot \beta\right)^{2}}{12 M_{\tilde{t}}^{2}}\right) .
\end{aligned}
$$

with $M_{\tilde{t}}^{2} \equiv m_{\tilde{t}_{1}} m_{\tilde{t}_{2}}$, and $g_{3}$ is the QCD coupling.

\subsection{The Higgs mass and mixing angle}

The scalars receive mass corrections from the usual one-loop radiative corrections but now also from the effective operators. Here we take the parameters $c_{0}, c_{1}, c_{2}$ to be real. We find the following result for the mass of the lightest Higgs scalar $h$ :

$$
m_{h}^{2}=\frac{1}{2}\left\{m_{A}^{2}+m_{Z}^{2}+\delta m_{Z}^{2} \sin ^{2} \beta-\sqrt{w}\right\}+\Delta m_{h}^{2}
$$

where

$$
w \equiv\left[\left(m_{A}^{2}-m_{Z}^{2}\right) \cos 2 \beta+\delta m_{Z}^{2} \sin ^{2} \beta\right]^{2}+\sin ^{2} 2 \beta\left(m_{A}^{2}+m_{Z}^{2}\right)^{2} .
$$

and where $\Delta m_{h}^{2}$ is the contribution due to the higher-dimensional operators:

$$
\Delta m_{h}^{2}=\left(2 \mu \frac{c_{0}}{M}\right) s_{1}+\left(2 \mu \frac{c_{0}}{M}\right)^{2} s_{2}+\left(\frac{g_{1}^{2} c_{1}}{M^{2}}+\frac{g_{2}^{2} c_{2}}{M^{2}}\right) s_{3}+\mathcal{O}\left(\frac{1}{M^{3}}\right)
$$

with

$$
\begin{aligned}
& s_{1}=v^{2} \sin 2 \beta\left\{1+\frac{\left(m_{A}^{2}+m_{Z}^{2}\right)}{\sqrt{w}}\right\} \\
& s_{2}=\frac{v^{4}}{4 \mu^{2}} \sin ^{2} 2 \beta+\frac{v^{4}}{\sqrt{w}}\left\{-1+\frac{1}{2 \mu^{2}}\left(m_{A}^{2}+m_{Z}^{2}\right) \sin ^{2} 2 \beta\right\}+\frac{1}{w^{3 / 2}}\left(m_{A}^{2}+m_{Z}^{2}\right)^{2} v^{4} \sin ^{2} 2 \beta \\
& s_{3}=\frac{v^{4}}{32} \sin 2 \beta+\frac{v^{4} \sin 2 \beta}{128 \sqrt{w}}\left[8 m_{A}^{2}-(4+3 \delta) m_{Z}^{2}+6 \delta m_{Z}^{2} \cos 2 \beta+3\left(4 m_{A}^{2}-\delta m_{Z}^{2}\right) \cos 4 \beta\right]
\end{aligned}
$$

where we kept (small) effects from the interplay between the effective operators and the one-loop correction to $V_{h}$. The mass of the CP-odd Higgs boson is

$$
m_{A}^{2}=\frac{2 B \mu}{\sin 2 \beta}-\frac{2 v^{2}}{\sin 2 \beta}\left(\frac{c_{0}}{M} \mu\right)-\frac{v^{4}}{32} \frac{\cos ^{2} 2 \beta}{\sin 2 \beta}\left(\frac{g_{1}^{2} c_{1}}{M^{2}}+\frac{g_{2}^{2} c_{2}}{M^{2}}\right)+\mathcal{O}\left(\frac{1}{M^{3}}\right)
$$


which does not receive one-loop corrections. The mixing angle $\alpha$ is given by

$$
\begin{gathered}
\tan 2 \alpha=-\frac{1}{\mathcal{D}}\left[\left(m_{A}^{2}+m_{Z}^{2}\right) \tan 2 \beta-\frac{2 v^{2}}{\cos 2 \beta}\left(2 \mu \frac{c_{0}}{M}\right)-\left(2 \frac{c_{0}}{M}\right)^{2} v^{4} \tan 2 \beta\right. \\
\left.+\left(\frac{g_{1}^{2} c_{1}}{M^{2}}+\frac{g_{2}^{2} c_{2}}{M^{2}}\right) \frac{v^{4}\left(m_{Z}^{2} f_{Z}-m_{A}^{2} f_{A}\right)}{32 \mathcal{D} \cos ^{2} 2 \beta}\right]
\end{gathered}
$$

with

$$
\begin{aligned}
\mathcal{D} & =m_{A}^{2}-m_{Z}^{2}+(\sec 2 \beta-1) \delta m_{Z}^{2} / 2 \\
f_{Z} & =4 \cos 2 \beta-(2-5 \cos 2 \beta+6 \cos 4 \beta-3 \cos 6 \beta) \delta / 4 \\
f_{A} & =\cos 2 \beta+3 \cos 6 \beta
\end{aligned}
$$

One can see the corrections to $\tan 2 \alpha$ due to the effective operators, that are used below.

We also note that the new operators correct the gauge field kinetic terms when the Higgs fields receive vevs. The corrected gauge couplings are the ones that appear in the following.

\section{Corrections to the partial widths of $h \rightarrow \gamma \gamma$ and $h \rightarrow Z \gamma$}

In this section we study how the new operators correct the Higgs couplings to the SM particles. To this end, we parametrize these corrections in terms of the usual MSSM Higgs couplings.

\subsection{Higgs couplings and signal rates}

The renormalizable part of the Lagrangian for the lightest neutral CP-even Higgs scalar $h$ can be written [22] as

$$
\begin{aligned}
\mathcal{L}_{\text {ren }}= & -c_{t} \frac{m_{t}}{v} h t \bar{t}-c_{c} \frac{m_{c}}{v} h c \bar{c}-c_{b} \frac{m_{b}}{v} h b \bar{b}-c_{\tau} \frac{m_{\tau}}{v} h \tau^{+} \tau^{-} \\
& +c_{Z} \frac{m_{Z}^{2}}{v} h Z^{\mu} Z_{\mu}+c_{W} \frac{2 m_{W}^{2}}{v} h W^{+\mu} W_{\mu}^{-}
\end{aligned}
$$

where the dimensionless coefficients are given by,

$$
c_{t}=c_{c}=\frac{\cos \alpha}{\sin \beta}, c_{b}=c_{\tau}=-\frac{\sin \alpha}{\cos \beta}, c_{Z}=c_{W}=\sin (\beta-\alpha) .
$$

where the mixing angle $\alpha$ is given in (2.14). In the scenario under consideration all loop corrections to the tree level coefficients in $(3.2)$ are negligible. The usual SM values for the couplings in (3.1) and (3.2) are obtained in the decoupling limit, in which $\alpha \rightarrow \beta-$ $\pi / 2$, implying that $\cos \alpha \rightarrow \sin \beta, \sin \alpha \rightarrow-\cos \beta$ and hence, $c_{i} \rightarrow c_{i}^{\mathrm{SM}}=1$, where $i=$ $t, c, b, \tau, Z, W$.

We work in the limit when loop contributions from superpartners and other Higgs scalars are negligible. The dimension-five part of the Higgs Lagrangian, which takes into account 1loop contributions from SM particles as well as the contributions from the effective operators 
in (2.4) can be written as

$$
\begin{aligned}
\mathcal{L}_{\operatorname{dim} 5}= & c_{g}^{\text {loop }} \frac{\alpha_{\mathrm{S}}}{12 \pi v} h \operatorname{Tr} G^{\mu \nu} G_{\mu \nu}+\left(c_{\gamma}^{\text {loop }}+c_{\gamma}^{\text {BMSSM }}\right) \frac{\alpha_{\mathrm{EM}}}{8 \pi v} h F^{\mu \nu} F_{\mu \nu} \\
& +\left(c_{\gamma Z}^{\text {loop }}+c_{\gamma Z}^{\mathrm{BMSSM}}\right) \frac{\alpha_{\mathrm{EM}}}{4 \pi \sin \theta_{w} v} h Z^{\mu \nu} F_{\mu \nu} .
\end{aligned}
$$

The 1-loop contributions to these coefficients are given by $\left[\begin{array}{l}4 \\ 23\end{array}\right.$

$$
\begin{aligned}
c_{g}^{\text {loop }} & =c_{t} \mathcal{A}_{g}^{(t)}+c_{b} \mathcal{A}_{g}^{(b)} \approx 1.03 c_{t}-(0.05+0.07 i) c_{b} \\
c_{\gamma}^{\text {loop }} & =c_{W} \mathcal{A}_{\gamma}^{(W)}+c_{t} \mathcal{A}_{\gamma}^{(t)} \approx-8.36 c_{W}+1.84 c_{t} \\
c_{Z \gamma}^{\text {loop }} & =c_{W} \mathcal{A}_{Z \gamma}^{(W)}+c_{t} \mathcal{A}_{Z \gamma}^{(t)} \approx 5.80 c_{W}-0.31 c_{t}
\end{aligned}
$$

where, in the last steps, we have inserted $m_{h}=126 \mathrm{GeV}$ in the 1-loop form factors $\mathcal{A}$, whose explicit expressions are given in appendix A.2. In the decoupling limit, where $c_{i} \rightarrow c_{i}^{\mathrm{SM}}=1$ in 3.2 , the $c^{\text {loop }}$-coefficients in 3.4 approach the values they have in the SM, which, for $m_{h}=126 \mathrm{GeV}$, follow trivially from $(3.4)$,

$$
\begin{aligned}
c_{g}^{\text {loop }} \rightarrow c_{g}^{\mathrm{SM}} & \approx 0.98+0.07 i \\
c_{\gamma}^{\text {loop }} \rightarrow c_{\gamma}^{\mathrm{SM}} & \approx-6.52 \\
c_{\gamma Z}^{\text {loop }} \rightarrow c_{\gamma Z}^{\mathrm{SM}} & \approx 5.49 .
\end{aligned}
$$

In order to obtain the $c_{\gamma}^{\mathrm{BMSSM}}$ and $c_{\gamma Z}^{\mathrm{BMSSM}}$ coefficients in 2.2 , we extract the following component interactions from the operators in 2.3,

$$
\begin{aligned}
\mathcal{O}_{6} & \supset-\sum_{s=1,2} \frac{c_{s}}{8 M^{2}} h_{u} \cdot h_{d}\left(F_{s}^{a \mu \nu} F_{s \mu \nu}^{a}+\frac{i}{2} \epsilon^{\mu \nu \rho \sigma} F_{s \mu \nu}^{a} F_{s \rho \sigma}^{a}\right)+\text { h.c. } \\
& \supset \frac{v \cos (\beta+\alpha)}{8 M^{2}}\left(\left[c_{1} \cos ^{2} \theta_{w}+c_{2} \sin ^{2} \theta_{w}\right] h F^{\mu \nu} F_{\mu \nu}+2\left(c_{2}-c_{1}\right) \sin \theta_{w} \cos \theta_{w} h F^{\mu \nu} Z_{\mu \nu}\right)
\end{aligned}
$$

where we have used,

$$
\begin{aligned}
h_{u} \cdot h_{d}=h_{u}^{+} h_{d}^{-}-h_{u}^{0} h_{d}^{0} \quad, \quad h_{i}^{0}=\frac{1}{\sqrt{2}}\left(v_{i}+\operatorname{Re} h_{i}^{0}+i \operatorname{Im} h_{i}^{0}\right), \\
\operatorname{Re} h_{d}^{0}=-\sin \alpha h+\cos \alpha H \quad, \quad \operatorname{Re} h_{u}^{0}=\cos \alpha h+\sin \alpha H, \\
A_{1 \mu}=\cos \theta_{w} A_{\mu}-\sin \theta_{w} Z_{\mu} \quad, \quad A_{2 \mu}^{(3)}=\sin \theta_{w} A_{\mu}+\cos \theta_{w} Z_{\mu}
\end{aligned}
$$

and $v_{d}=v \cos \beta, v_{u}=v \sin \beta$, with $v=246 \mathrm{GeV}$. Moreover, the hypercharge gauge boson $A_{1 \mu}$ and the (third component of the) $\mathrm{SU}(2)_{L}$ gauge boson $A_{2 \mu}^{(3)}$ have been rewritten in terms of the photon $A_{\mu}$ and the $Z$ boson $Z_{\mu}$. Note that there is also a dimension 5 operator generated from (2.3) that involves the Higgs scalar $h$ and two field strengths of the $Z$ boson (as well as an analogous operator involving two field strengths of the $W$ boson). However, since these operators will have couplings comparable to the $\gamma \gamma$ or $Z \gamma$ couplings, but strongly

\footnotetext{
${ }^{4}$ See also [24] for additional studies of $h \rightarrow Z \gamma$.
} 
phase space suppressed, we expect them to be irrelevant with respect to the usual dimension 3 Higgs coupling to the $Z$ and $W$ bosons in (3.1). Therefore, we do not consider them.

The contributions to 3.3 from $(3.6)$ are given by:

$$
\begin{aligned}
c_{\gamma}^{\mathrm{BMSSM}} & =\frac{\pi v^{2} \cos (\beta+\alpha)}{M^{2} \alpha_{\mathrm{EM}}}\left(c_{1} \cos ^{2} \theta_{w}+c_{2} \sin ^{2} \theta_{w}\right) \\
c_{\gamma Z}^{\mathrm{BMSSM}} & =\frac{\pi v^{2} \cos (\beta+\alpha)}{M^{2} \alpha_{\mathrm{EM}}}\left(c_{2}-c_{1}\right) \sin ^{2} \theta_{w} \cos \theta_{w} .
\end{aligned}
$$

In the decoupling limit, where $\cos (\beta+\alpha) \rightarrow \sin 2 \beta$, we see that the coefficients in (3.8) are maximized for small $\tan \beta$.

We can now define the relevant Higgs partial decay widths, normalized to the corresponding SM value, in terms of the dimensionless $c$-coefficients in (3.2), (3.4), (3.5), (3.8),

$$
\frac{\Gamma_{h i i}}{\Gamma_{h i i}^{\mathrm{SM}}}=\left|c_{i}\right|^{2}, \frac{\Gamma_{h g g}}{\Gamma_{h g g}^{\mathrm{SM}}}=\left|\frac{c_{g}^{\text {loop }}}{c_{g}^{\mathrm{SM}}}\right|^{2}, \frac{\Gamma_{h \gamma \gamma}}{\Gamma_{h \gamma \gamma}^{\mathrm{SM}}}=\left|\frac{c_{\gamma}^{\text {loop }}+c_{\gamma}^{\mathrm{BMSSM}}}{c_{\gamma}^{\mathrm{SM}}}\right|^{2}, \frac{\Gamma_{h \gamma Z}}{\Gamma_{h \gamma Z}^{\mathrm{SM}}}=\left|\frac{c_{\gamma Z}^{\text {loop }}+c_{\gamma Z}^{\mathrm{BMSSM}}}{c_{\gamma Z}^{\mathrm{SM}}}\right|^{2}
$$

as well as the corresponding branching ratios (BRs),

$$
\begin{gathered}
\frac{\mathrm{BR}_{h i i}}{\mathrm{BR}_{h i i}^{\mathrm{SM}}}=\left|\frac{c_{i}}{c_{\text {tot }}}\right|^{2}, \frac{\mathrm{BR}_{h g g}}{\mathrm{BR}_{h g g}^{\mathrm{SM}}}=\left|\frac{c_{g}^{\text {loop }}}{c_{g}^{\mathrm{SM}} c_{\text {tot }}}\right|^{2} \\
\frac{\mathrm{BR}_{h \gamma \gamma}}{\mathrm{BR}_{h \gamma \gamma}^{\mathrm{SM}}}=\left|\frac{c_{\gamma}^{\text {loop }}+c_{\gamma}^{\mathrm{BMSSM}}}{c_{\gamma}^{\mathrm{SM}} c_{\text {tot }}}\right|^{2} \quad, \quad \frac{\mathrm{BR}_{h \gamma Z}}{\mathrm{BR}_{h \gamma Z}^{\mathrm{SM}}}=\left|\frac{c_{\gamma Z}^{\text {loop }}+c_{\gamma Z}^{\mathrm{BMSSM}}}{c_{\gamma Z}^{\mathrm{SM}} c_{\mathrm{tot}}}\right|^{2} .
\end{gathered}
$$

The coefficient $c_{\text {tot }}$ in 3.10 can be written as,

$$
\left|c_{\text {tot }}\right|^{2}=\sum_{i=t, c, b, \tau, Z, W}\left|c_{i}\right|^{2} \mathrm{BR}_{h i i}^{\mathrm{SM}}+\left|\frac{c_{g}^{\text {loop }}}{c_{g}^{\mathrm{SM}}}\right|^{2} \mathrm{BR}_{h g g}^{\mathrm{SM}}
$$

where we have neglected the contributions from, for example, $h \rightarrow \gamma \gamma$ and $h \rightarrow Z \gamma$, as well as possible invisible decays. Let us now define the inclusive, as well as the individual gluongluon fusion ( $\mathrm{ggF})$, vector boson fusion $(\mathrm{VBF})$ and vector boson associated $(\mathrm{VH})$ production cross sections, normalized with respect to the corresponding SM values,

$$
\begin{aligned}
& \frac{\sigma_{\mathrm{incl}}}{\sigma_{\text {incl }}^{\mathrm{SM}}}=\frac{\left|c_{g}^{\text {loop }} / c_{g}^{\mathrm{SM}}\right|^{2} \sigma_{\mathrm{ggF}}^{\mathrm{SM}}+\left|c_{V}\right|^{2}\left(\sigma_{\mathrm{VBF}}^{\mathrm{SM}}+\sigma_{\mathrm{VH}}^{\mathrm{SM}}\right)}{\sigma_{\mathrm{ggF}}^{\mathrm{SM}}+\sigma_{\mathrm{VBF}}^{\mathrm{SM}}+\sigma_{\mathrm{VH}}^{\mathrm{SM}}}, \\
& \frac{\sigma_{\mathrm{ggF}}}{\sigma_{\mathrm{ggF}}^{\mathrm{SM}}}=\left|c_{g}^{\text {loop }} / c_{g}^{\mathrm{SM}}\right|^{2}, \frac{\sigma_{\mathrm{VBF}}}{\sigma_{\mathrm{VBF}}^{\mathrm{SM}}}=\frac{\sigma_{\mathrm{VH}}}{\sigma_{\mathrm{VH}}^{\mathrm{SM}}}=\left|c_{V}\right|^{2}
\end{aligned}
$$

where we have denoted $c_{V}=c_{Z}=c_{W}$, since the Higgs couplings to $Z$ and $W$ bosons coincide in 3.2 . We can now write, for example, the signal rates in the inclusive and dijet channels 
of the $h \rightarrow \gamma \gamma$ decay mode, again normalized with respect to the SM,

$$
\begin{aligned}
R_{\gamma \gamma}^{\text {incl }} & =\frac{\sigma_{\mathrm{tot}}}{\sigma_{\mathrm{tot}}^{\mathrm{SM}}} \frac{\mathrm{BR}_{h \gamma \gamma}}{\mathrm{BR}} \mathrm{R}_{h \gamma}^{\mathrm{SM}} \\
R_{\gamma \gamma}^{\mathrm{dijet}} & =\frac{\epsilon_{\mathrm{ggF}}^{\gamma}\left|c_{g}^{\text {loop }} / c_{g}^{\mathrm{SM}}\right|^{2} \sigma_{\mathrm{ggF}}^{\mathrm{SM}}+\epsilon_{\mathrm{VBF}}^{\gamma}\left|c_{V}\right|^{2} \sigma_{\mathrm{VBF}}^{\mathrm{SM}}+\epsilon_{\mathrm{VH}}^{\gamma}\left|c_{V}\right|^{2} \sigma_{\mathrm{VH}}^{\mathrm{SM}}}{\epsilon_{\mathrm{ggF}}^{\gamma} \sigma_{\mathrm{ggF}}^{\mathrm{SM}}+\epsilon_{\mathrm{VBF}}^{\gamma} \sigma_{\mathrm{VBF}}^{\mathrm{SM}}+\epsilon_{\mathrm{VH}}^{\gamma} \sigma_{\mathrm{VH}}^{\mathrm{SM}}} \frac{\mathrm{BR}_{h \gamma \gamma}^{\mathrm{SM}}}{}
\end{aligned}
$$

where the $\epsilon^{\gamma}$-coefficients are the selection efficiencies for the different production modes in the dijet-tag category of final states.

\subsection{The decoupling limit}

Let us now take the decoupling limit, in which,

$$
\frac{c_{i}}{c_{i}^{\mathrm{SM}}}=\frac{c_{g}^{\text {loop }}}{c_{g}^{\mathrm{SM}}}=\frac{c_{\gamma}^{\text {loop }}}{c_{\gamma}^{\mathrm{SM}}}=\frac{c_{\gamma Z}^{\text {loop }}}{c_{\gamma Z}^{\mathrm{SM}}}=1
$$

where $i=t, c, b, \tau, Z, W$. This implies that $\left|c_{\text {tot }}\right|=1$ in (3.11) and that,

$$
\frac{\Gamma_{h i i}}{\Gamma_{h i i}^{\mathrm{SM}}}=\frac{\mathrm{BR}_{h i i}}{\mathrm{BR}_{h i i}^{\mathrm{SM}}}=\frac{\Gamma_{h g g}}{\Gamma_{h g g}^{\mathrm{SM}}}=\frac{\mathrm{BR}_{h g g}}{\mathrm{BR}_{h g g}^{\mathrm{SM}}}=1
$$

whereas,

$$
\frac{\Gamma_{h \gamma \gamma}}{\Gamma_{h \gamma \gamma}^{\mathrm{SM}}}=\frac{\mathrm{BR}_{h \gamma \gamma}}{\mathrm{BR}_{h \gamma \gamma}^{\mathrm{SM}}}=\left|1+\frac{c_{\gamma, \mathrm{dec}}^{\mathrm{BMSM}}}{c_{\gamma}^{\mathrm{SM}}}\right|^{2} \quad, \frac{\Gamma_{h \gamma Z}}{\Gamma_{h \gamma Z}^{\mathrm{SM}}}=\frac{\mathrm{BR}_{h \gamma Z}}{\mathrm{BR}_{h \gamma Z}^{\mathrm{SM}}}=\left|1+\frac{c_{\gamma Z, \mathrm{dec}}^{\mathrm{BMSM}}}{c_{\gamma Z}^{\mathrm{SM}}}\right|^{2}
$$

for which the coefficients in (3.8) are given by, in the decoupling limit,

$$
\begin{aligned}
c_{\gamma, \text { dec }}^{\mathrm{BMSSM}} & =\frac{\pi v^{2} \sin 2 \beta}{M^{2} \alpha_{\mathrm{EM}}}\left(c_{1} \cos ^{2} \theta_{w}+c_{2} \sin ^{2} \theta_{w}\right) \\
c_{\gamma Z, \mathrm{dec}}^{\mathrm{BMSSM}} & =\frac{\pi v^{2} \sin 2 \beta}{M^{2} \alpha_{\mathrm{EM}}}\left(c_{2}-c_{1}\right) \sin ^{2} \theta_{w} \cos \theta_{w} .
\end{aligned}
$$

In the decoupling limit, the production cross sections in 3.12 are all equal to their SM corresponding SM value,

$$
\frac{\sigma_{\mathrm{incl}}}{\sigma_{\mathrm{incl}}^{\mathrm{SM}}}=\frac{\sigma_{\mathrm{ggF}}}{\sigma_{\mathrm{ggF}}^{\mathrm{SM}}}=\frac{\sigma_{\mathrm{VBF}}}{\sigma_{\mathrm{VBF}}^{\mathrm{SM}}}=\frac{\sigma_{\mathrm{VH}}}{\sigma_{\mathrm{VH}}^{\mathrm{SM}}}=1
$$

Thus, all signal rates, for any production mode, associated with the channels $h \rightarrow i$, for $i=t, c, b, \tau, Z, W$, as well as $h \rightarrow g g$, will be equal to their corresponding SM value. In the $h \rightarrow \gamma \gamma$ channel, we see that the signal rates in (3.13) (as well as any other signal rate in the $h \rightarrow \gamma \gamma$ channel) will be given by the corresponding normalized partial width,

$$
R_{\gamma \gamma}=R_{\gamma \gamma}^{\mathrm{incl}}=R_{\gamma \gamma}^{\mathrm{dijet}}=\frac{\Gamma_{h \gamma \gamma}}{\Gamma_{h \gamma \gamma}^{\mathrm{SM}}}=\left|1+\frac{c_{\gamma, \mathrm{dec}}^{\mathrm{BMSM}}}{c_{\gamma}^{\mathrm{SM}}}\right|^{2}
$$


and the same for the $h \rightarrow Z \gamma$ channel,

$$
R_{Z \gamma}=R_{Z \gamma}^{\mathrm{incl}}=R_{Z \gamma}^{\mathrm{dijet}}=\frac{\Gamma_{h Z \gamma}}{\Gamma_{h Z \gamma}^{\mathrm{SM}}}=\left|1+\frac{c_{Z \gamma, \mathrm{dec}}^{\mathrm{BMSSM}}}{c_{Z \gamma}^{\mathrm{SM}}}\right|^{2}
$$

In summary, in the decoupling limit, all the partial decay widths, except for $\Gamma_{h \gamma \gamma}$ and $\Gamma_{h Z \gamma}$, are all equal to their corresponding SM value. This implies that all the production cross sections, as well as the signal rates in all other channels, are equal to their SM values. Moreover, as seen in (3.19) and (3.20), the partial decay widths for $h \rightarrow \gamma \gamma$ and $h \rightarrow Z \gamma$, normalized with respect to the SM values, coincide with the corresponding signal rates. Hence, in this limit, $\Gamma_{h \gamma \gamma} / \Gamma_{h \gamma \gamma}^{\mathrm{SM}}$ and $\Gamma_{h Z \gamma} / \Gamma_{h Z \gamma}^{\mathrm{SM}}$ can be compared directly to the measured signal rates. From (3.17) notice that if $c_{1}=c_{2}$ one can change $\Gamma_{h \gamma \gamma}$ without affecting $\Gamma_{h Z \gamma}$.

\section{Results}

We can now evaluate the effect of the operators in 2.2 and 2.3 on the mass of the lightest neutral CP-even Higgs particle $h$ and on the partial decay widths $\Gamma_{h \gamma \gamma}$ and $\Gamma_{h Z \gamma}$, that directly correspond to the rates in the decoupling limit, as discussed in the previous section.

The Higgs mass in 2.9 as a function of $\tan \beta$ is displayed in figure 1. It is well known
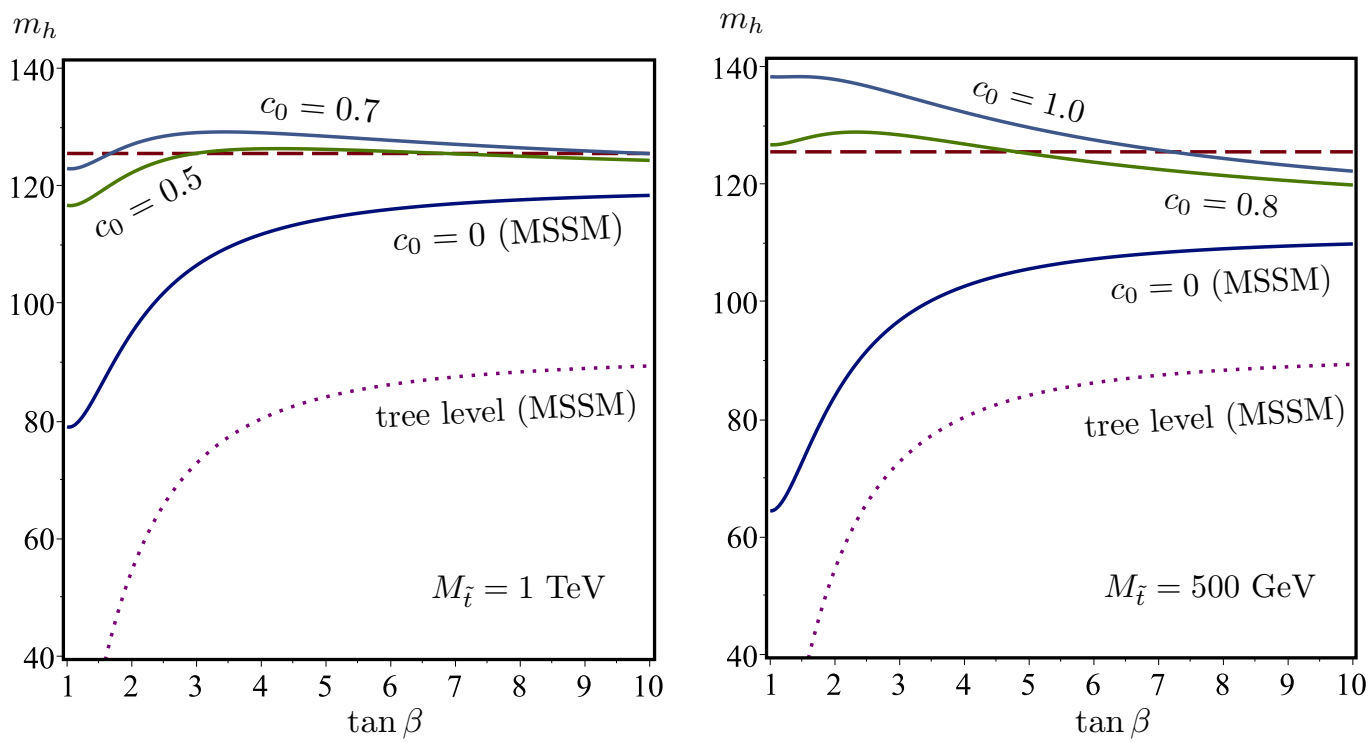

Figure 1: The mass of the lightest Higgs particle $h$ as a function of $\tan \beta$, for $M=5$ $\mathrm{TeV}, \mu=300 \mathrm{GeV}, m_{A}=1 \mathrm{TeV}$, no mixing $\left(X_{t}=0\right), M_{\tilde{t}}=500 \mathrm{GeV}$ (left panel) and $M_{\tilde{t}}=1 \mathrm{TeV}$ (right panel), and for various values of the $\mathcal{O}_{5}$ coefficient $c_{0}$. The solid curves include the MSSM loop corrections.

that we can accommodate a Higgs mass at $126 \mathrm{GeV}$ by tuning the soft parameters in the loop correction (2.7), but this usually demands a large $\tan \beta$, which we do not consider here 

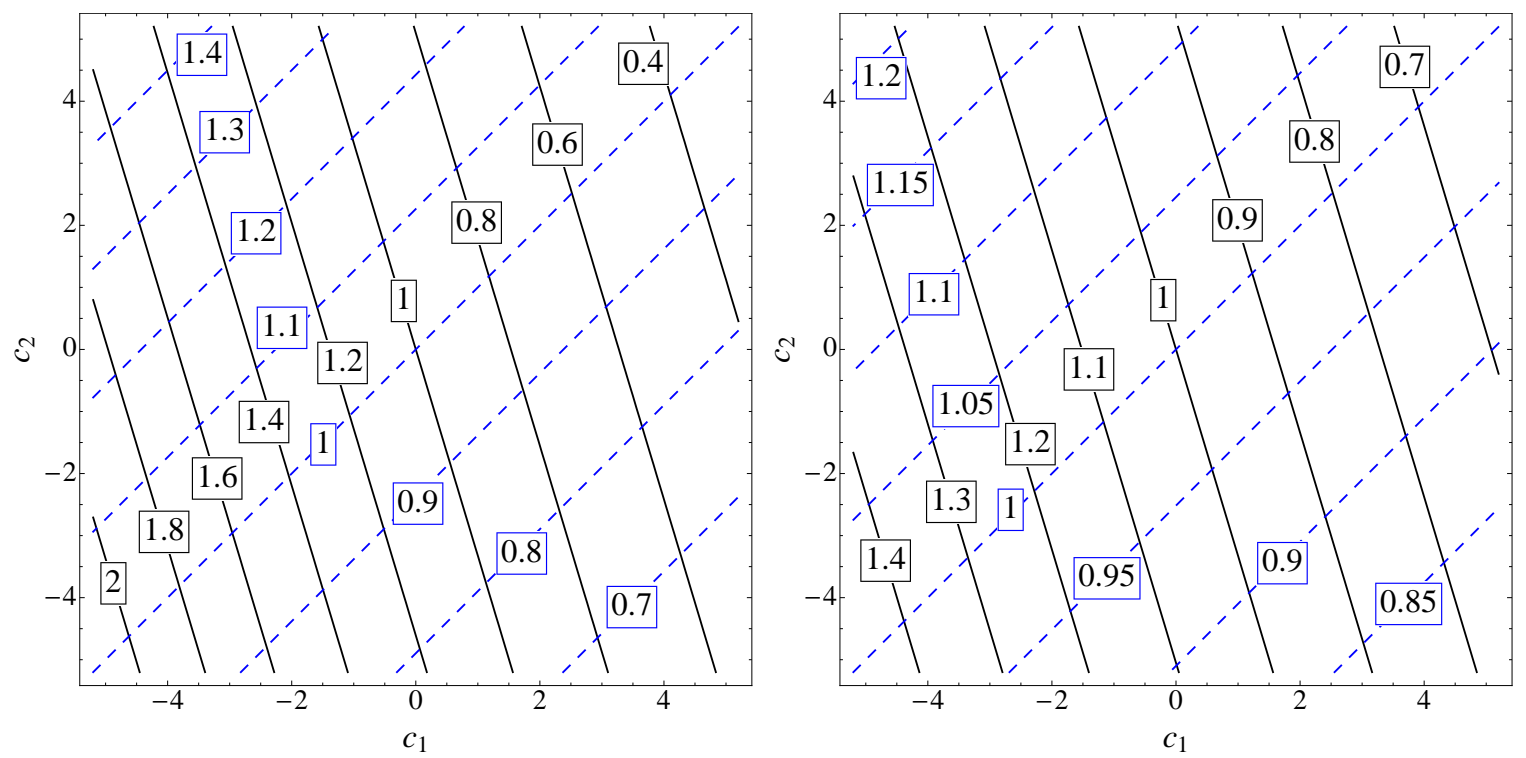

Figure 2: The $h \rightarrow \gamma \gamma$ signal rate $R_{\gamma \gamma}$ (solid black lines) in (3.19) and the $h \rightarrow Z \gamma$ rate $R_{Z \gamma}$ (dashed blue lines) in (3.20) are shown as functions of the coefficients $c_{1}$ and $c_{2}$ of the operators in $(2.2)$ and (2.3). In the plots we have set $\tan \beta=3$ (left panel) and $\tan \beta=7$ (right panel), $M=5 \mathrm{TeV}$ and taken the decoupling limit.

(since then additional Yukawa couplings that we do not include become important). With the $d=5$ operator 2.2 , one can easily obtain a value of $m_{h} \approx 126 \mathrm{GeV}$, see figure 1 ; this has an acceptable fine tuning $\Delta<30$ [13], even for small $\tan \beta<10$, which is an otherwise very fine-tuned region of the MSSM. Therefore the effect of the $d=5$ operator is more important than usually thought.

The dimensionless parameter $\epsilon \equiv c_{0} \mu / M$ measures the extent to which the contribution from $\mathcal{O}_{5}$ to the mass can be considered perturbative, and for the given numbers it is below $\epsilon<0.06$. The mass contributions from $\mathcal{O}_{6}$ rarely affect noticeably the curves in figure 1, but they are included for completeness (in the figure, $c_{1}=c_{2}=-1$ ). We included only a subset of the loop corrections, that is relevant at low $\tan \beta$, so we expect the curves to differ from the complete result by few $\mathrm{GeV}$ only, which we confirmed in our examples using FeynHiggs [25].

For the value of the scale $M$ found above in the region of $5 \mathrm{TeV}$, not within the LHC reach, one would like to examine the signal rates for $h \rightarrow \gamma \gamma$ and $h \rightarrow Z \gamma$. These signal rates $R_{\gamma \gamma}$ and $R_{Z \gamma}$ in the decoupling limit, given in eqs. (3.19) and (3.20), are shown in figure 2 as functions of the coefficients $c_{1}$ and $c_{2}$ of the operators in $(2.2)$ and $(2.3)$. Concerning the

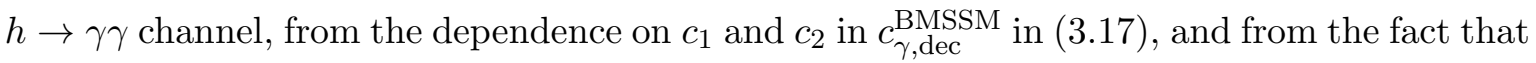
$c_{\gamma}^{\mathrm{SM}}$ is negative in (3.5), we see that the maximal enhancement of $R_{\gamma \gamma}$ in 3.19$)$ is obtained for negative values of both $c_{1}$ and $c_{2}$. In contrast, for positive (and not too large) values of the two coefficients, the $h \rightarrow \gamma \gamma$ signal is depleted with respect to the SM prediction, as can 

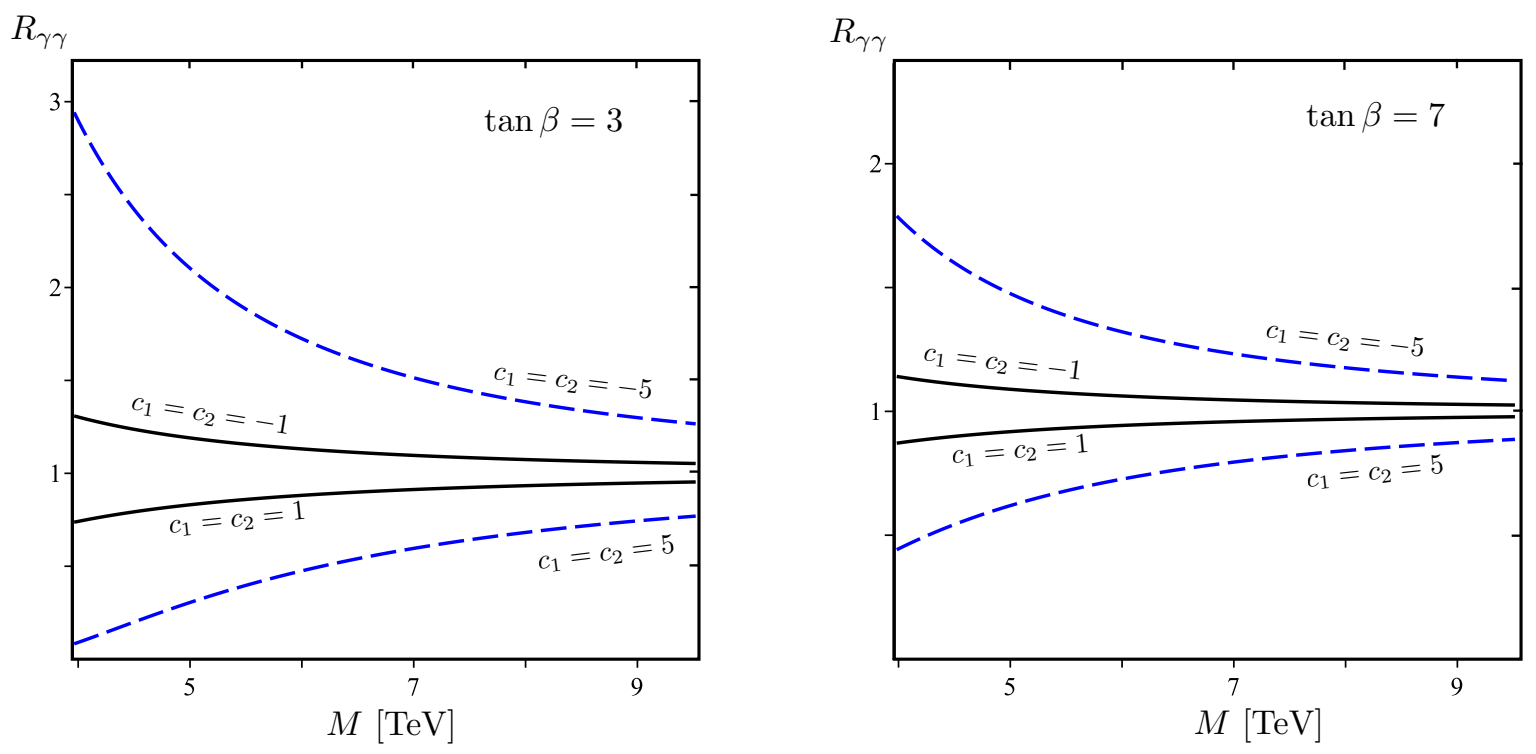

Figure 3: The $h \rightarrow \gamma \gamma$ signal rate $R_{\gamma \gamma}$ from (3.19) as functions of the scale $M$, where we have set the coefficients of the two operators in $(2.3)$ equal, $c_{1}=c_{2}$. We have set $M_{\tilde{t}}=1 \mathrm{TeV}, \mu=300 \mathrm{GeV}, m_{A}=1 \mathrm{TeV}, X_{t}=0$. Dashed blue lines provide rough estimates of the range of validity of the effective field theory.

be seen in figure 2. We emphasize again that we actually do not need both coefficients $c_{1}$ and $c_{2}$ (corresponding to the $\mathrm{U}(1)_{Y}$ and $\mathrm{SU}(2)_{L}$ operators in (2.3) ) to achieve enhancement, we could set e.g. $c_{2}=0$, but the flexibility this additional parameter affords is useful in the next figure. The maximum allowed enhancement continues to decrease for larger values of $\tan \beta$, unless of course if we simultaneously lower $M$.

From (3.17) we see that $c_{\gamma Z \text {,dec }}^{\mathrm{BMSSM}}$ is maximized when $c_{1}$ and $c_{2}$ have opposite signs. Moreover, since $c_{\gamma Z}^{\mathrm{SM}}$ is positive in (3.5), in order to achieve an enhancement of $R_{Z \gamma}$ in (3.19), it is required that $\left(c_{2}-c_{1}\right)>0$. This is seen in figure 2 , where $R_{Z \gamma}$ is maximized for large positive values for $c_{2}$ and large negative values for $c_{1}$. Notice that the dependence on the sign of $c_{1}$ and $c_{2}$ for $R_{\gamma \gamma}$ and $R_{Z \gamma}$ is not specific to this scenario or SUSY. It simply follows from EW symmetry breaking, as can be seen in (3.6).

In figures 3 and 4 , we show a different representation of the same physics as in figure 2 , where we fix the coefficients $c_{1}$ and $c_{2}$ in each curve, and instead vary the overall scale of new physics $M$. As expected, the effect of the higher-dimensional operators decreases with increasing $M$, but even for $M$ approaching $10 \mathrm{TeV}$ there can be some small effect. This perhaps somewhat counter-intuitive behavior is simply because the relevant SM couplings are small to begin with, as emphasized in the introduction. Since the "new physics" that generated these operators comes from a scale around or larger than $5 \mathrm{TeV}$, it will not be within easy reach of the LHC. 
In figure 4 we have illustrated the behavior of $R_{\gamma Z}$ if we require for example $R_{\gamma \gamma}=1.4$ (i.e. interpret the diphoton excess as signal) and vary $-5<c_{1}<5$, so each curve represents a particular value of $c_{2}$, and ends at some upper bound value of $M$ where it is no longer possible to achieve the prescribed value of $R_{\gamma \gamma}=1.4$. We see that above $\tan \beta=5$ or so, one would have to rely on the scale $M$ being not too far above $5 \mathrm{TeV}$.
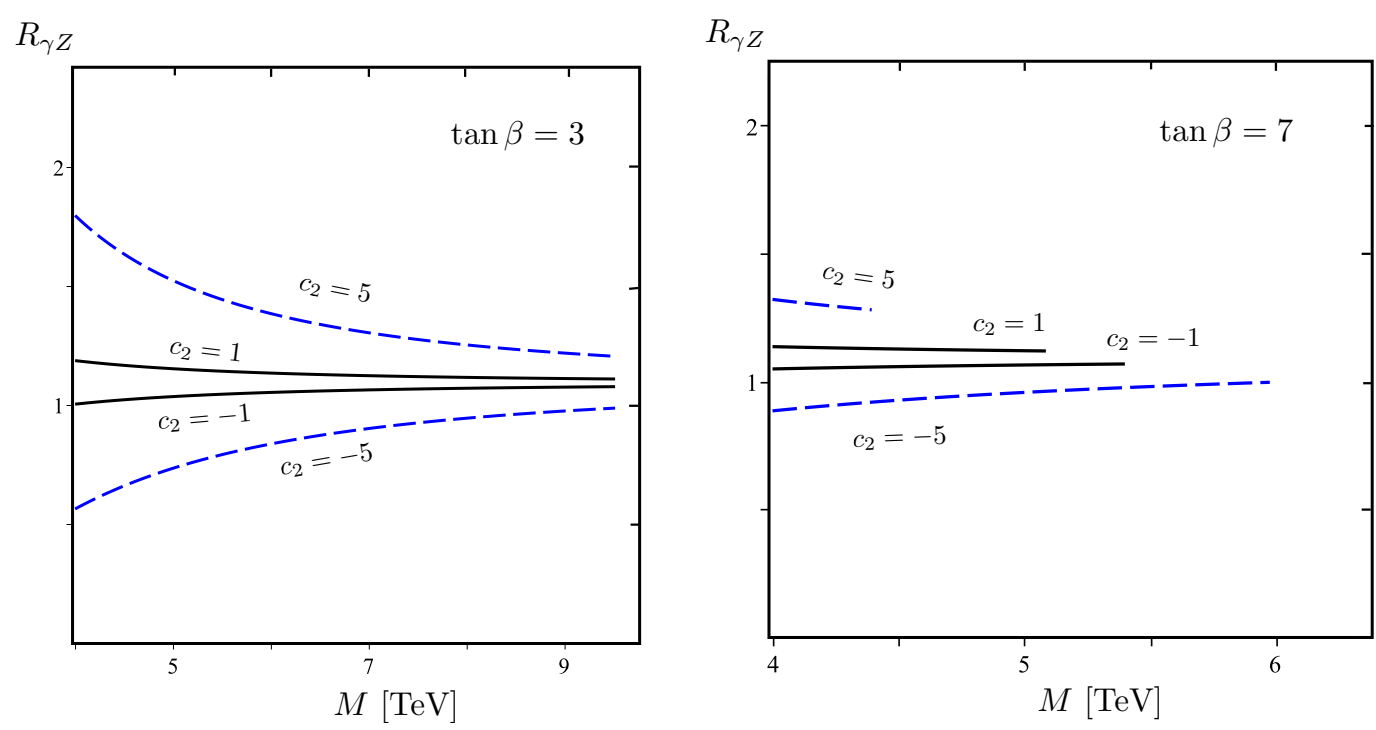

Figure 4: The $h \rightarrow Z \gamma$ rate $R_{Z \gamma}$ from (3.20) for various values of the coefficients $c_{1}$ and $c_{2}$ of the operators in (2.2) and (2.3), if we require $R_{\gamma \gamma}=1.4$ and vary $-5<c_{1}<5$ for fixed $c_{2}$. The curve ends when $c_{1}$ goes out of range for $R_{\gamma \gamma}=1.4$ with the given parameters. We have set $M_{\tilde{t}}=1 \mathrm{TeV}, \mu=300 \mathrm{GeV}, m_{A}=1 \mathrm{TeV}$, $X_{t}=0$. Dashed blue lines provide rough estimates of the range of validity of the effective field theory expansion.

With hindsight, it may appear that the analysis of the effects of $\mathcal{O}_{5}$ in $(2.2)$ and $\mathcal{O}_{6}$ in (2.3) could have been performed mostly independently of each other. To be clear, we did not assume this: as a matter of principle, we always include the contribution to e.g. the mixing angle $\alpha$ from $(2.2)$ when computing the effects of (2.3). But we emphasize that the $R_{\gamma \gamma}$ contributions arising from the dimension-six operators in 2.3 are maximized for small $\tan \beta$, see (3.17). Therefore, since the usual MSSM tree level contribution to the Higgs mass is minimized for small $\tan \beta$, the contribution from the dimension-five operator to the tree level Higgs mass is crucial in order to accommodate a $126 \mathrm{GeV}$ Higgs mass, as is seen in figure 1 .

\section{Generating the effective operators from underlying physics}

The natural question is then what "new physics" could generate the effective operators discussed. In this section we discuss a simple example of an underlying model from which both 
$\mathcal{O}_{5}$ and $\mathcal{O}_{2}$ arise simultaneously in the low energy effective theory, upon integrating out some massive supersymmetric degrees of freedom. Consider a model that contains the following superpotential, involving a massive gauge singlet chiral superfield ${ }^{5} \Sigma$ :

$$
W \supset(\mu+\lambda \Sigma) H_{d} \cdot H_{u}+\frac{1}{2} \mu_{S} \Sigma^{2}
$$

and a gauge kinetic function $\tau$ that depends on $\Sigma$,

$$
\tau(\Sigma) \operatorname{Tr}\left(W_{\alpha} W^{\alpha}\right) \quad \text { with } \quad \tau(\Sigma) \supset \frac{\Sigma}{\Lambda}
$$

where $\Lambda$ is a dimensionful suppression scale. If the SUSY mass $\mu_{S}$ is sufficiently large with respect to the energy scale under consideration, then $\Sigma$ can be integrated out supersymmetrically via its holomorphic equation of motion, which sets

$$
\Sigma=-\frac{\lambda}{\mu_{S}} H_{d} \cdot H_{u}-\frac{1}{\mu_{S} \Lambda} \operatorname{Tr}\left(W_{\alpha} W^{\alpha}\right)+\cdots
$$

where the dots stand for higher dimensional terms (further suppressed by $\mu_{S}, \Lambda$ ). By inserting this solution back into the original Lagrangian, we obtain the following terms,

$$
\begin{aligned}
& \int d^{2} \theta\left(\mu H_{d} \cdot H_{u}-\frac{\lambda^{2}}{2 \mu_{S}}\left(H_{d} \cdot H_{u}\right)^{2}-\frac{\lambda}{\mu_{S} \Lambda} H_{d} \cdot H_{u} \operatorname{Tr}\left(W_{\alpha} W^{\alpha}\right)\right) \\
& +\int d^{4} \theta\left(\left|\frac{\lambda}{\mu_{S}}\right|^{2}\left(H_{d} \cdot H_{u}\right)^{\dagger}\left(H_{d} \cdot H_{u}\right)\right)
\end{aligned}
$$

where we have included operators up to dimension-six. We see that operators $\mathcal{O}_{5}, \mathcal{O}_{6}$ of (2.2), (2.3) were simultaneously generated as a consequence of integrating out $\Sigma$.

The dimension-six Kähler potential operator in the second line of (5.4) gives corrections to the quartic Higgs scalar potential, and hence to the tree level Higgs mass. However, in comparison to our dimension-five operator in the first line of (5.4), this operator is suppressed by a higher power of $\mu_{S}$. As long as $\mu_{S}$ is sufficiently large, the corrections from this dimension-six operator will be smaller in size with respect to the corrections from the dimension-five operator.

It should be acknowledged that this example is not renormalizable since the gauge kinetic term (5.2) has dimension $d=5$. To have a renormalizable microscopic model, one should also specify the degrees of freedom responsible for generating this $d=5$ operator. Nevertheless this operator with a moduli-dependent gauge kinetic function is generically present in models derived from supergravity or string theory.

Finally in order to connect with the discussion in the rest of the paper, we should assume that the dimension-five operators in $(5.2)$ only involve the $\mathrm{U}(1)_{Y}$ and $\mathrm{SU}(2)_{L}$ gauge field strength, and not also the $\mathrm{SU}(3)_{C}$ one that could in principle be also present. In string models, something along these lines could be achieved by for example considering a brane

\footnotetext{
${ }^{5}$ Gauge singlet fields with a supersymmetric mass term appear in general versions of the NMSSM [13, 14, 12].
} 
model in which the dimensionality of the branes that give rise to the $\mathrm{U}(1)_{Y}$ and $\mathrm{SU}(2)_{L}$ gauge fields is different from those that give rise to the $\mathrm{SU}(3)_{C}$ gauge field. In this way, since the $\mathrm{U}(1)_{Y}$ and $\mathrm{SU}(2)_{L}$ branes and the $\mathrm{SU}(3)_{C}$ branes, respectively, wrap different cycles of the internal geometry, they depend on different gauge singlet moduli fields associated with the different cycles.

\section{Conclusions}

Recent LHC data on the Higgs mass and its couplings to the photon, and the negative SUSY searches, present increasing difficulties for MSSM-like models to naturally accommodate a Higgs mass near $126 \mathrm{GeV}$, without undue fine-tuning, and a potential enhancement of the $h \rightarrow \gamma \gamma$ partial decay rate, without affecting other partial decay widths. Motivated by these observations, in this work we investigated whether supersymmetric effects beyond the MSSM (and even beyond reach for the LHC), could simultaneously accommodate these results. Using an effective approach, we identified two effective operators of dimensions $d=5$ and $d=6$ that can address these problems and give the leading order corrections to the Higgs quartic coupling and the Higgs coupling to photons, respectively.

We showed that the MSSM with small, supersymmetric corrections due to these effective operators can simultaneously naturally accommodate a Higgs boson with a mass near 126 $\mathrm{GeV}$, an enhanced Higgs coupling to photons (and also $Z \gamma$ ) relative to the SM expectation, and finally with SM-like Higgs couplings to the other SM particles. The scale of the supersymmetric effective operators is in the region of $5 \mathrm{TeV}$ or even larger, and therefore possibly not within the LHC reach. The corrections from the dimension-six operators to the Higgs coupling to photons (and $Z \gamma$ ) are maximized for small $\tan \beta$ which is also the region where the dimension-five operator produces the most dramatic effect relative to the MSSM. This suggests that it is natural to consider these operators together and this is further supported by the fact that both of them can be generated simultaneously by an underlying model, as we showed.

There remains the question of how the existence of these operators can be tested. Let us assume that the signal rate in the $h \rightarrow \gamma \gamma$ channel is confirmed to be higher than the SM expectation while the signal rates in all the other channels coincide with the SM values. If at the same time, one can rule out light stau sleptons in the mass range (of $150 \mathrm{GeV}$ or so), needed in order to enhance the diphoton signal with the correct amount, this would cause a real problem for the MSSM and physics beyond the MSSM will be required. Should the excess go away when further data is analyzed, our results will remain useful to provide bounds on the scale $M$ of supersymmetric "new physics" beyond the MSSM. Either way, this suggests that the diphoton rate is a useful, sensitive probe in this context. 


\section{Acknowledgements}

The work of C. Petersson is supported in part by IISN-Belgium (conventions 4.4511.06, 4.4505.86 and 4.4514.08), by the "Communauté Française de Belgique" through the ARC program and by a "Mandat d'Impulsion Scientifique" of the F.R.S.-FNRS. The work of D. M. Ghilencea was supported by a grant of the Romanian National Authority for Scientific Research, CNCS - UEFISCDI, project number PN-II-ID-PCE-2011-3-0607.

\section{A Appendix}

\section{A.1 Details concerning the Lagrangian}

Here we derive the Lagrangian of Section 2. Unlike in the text, we also include SUSY breaking effects associated with operators $\mathcal{O}_{5}, \mathcal{O}_{6}$, by using the spurion field. The starting point is

$$
\mathcal{L}=\int d^{4} \theta \sum_{i=u, d}\left(1-m_{i}^{2} \theta^{2} \bar{\theta}^{2}\right) H_{i}^{\dagger} e^{V_{i}} H_{i}+\left\{\int d^{2} \theta \mu\left(1+B \theta^{2}\right) H_{d} \cdot H_{u}+\text { h.c. }\right\}+\mathcal{O}_{5}+\mathcal{O}_{6}
$$

The superfield components are $V_{i}=\left(\lambda_{i}, V_{i, \mu}, D_{i}^{a} / 2\right), H_{i}=\left(h_{i}, \psi_{i}, F_{i}\right)$. Also $H_{d} \cdot H_{u}=\epsilon^{i j} H_{d}^{i} H_{u}^{j}$, with $\epsilon^{i j} \epsilon^{k j}=\delta^{i k} ; \epsilon^{i j} \epsilon^{k l}=\delta^{i k} \delta^{j l}-\delta^{i l} \delta^{j k}, \epsilon^{12}=1, h_{d} \cdot h_{u}=h_{d}^{0} h_{u}^{0}-h_{d}^{-} h_{u}^{+}$. Further

$$
\begin{aligned}
\mathcal{O}_{5} & =\frac{1}{M} \int d^{2} \theta\left(c_{0}+c_{0}^{\prime} \theta^{2}\right)\left(H_{u} \cdot H_{d}\right)^{2}+\text { h.c. } \\
& =\frac{c_{0}}{M}\left[2\left(h_{u} \cdot h_{d}\right)\left(h_{u} \cdot F_{d}+F_{u} \cdot h_{d}-\psi_{u} \cdot \psi_{d}\right)-\left(h_{u} \cdot \psi_{d}+\psi_{u} \cdot h_{d}\right)^{2}\right]+\frac{c_{0}^{\prime}}{M}\left(h_{u} \cdot h_{d}\right)^{2}+\text { h.c. }
\end{aligned}
$$

and

$$
\begin{aligned}
\mathcal{O}_{6} & =\frac{1}{M^{2}} \sum_{s=1,2} \frac{1}{16 g_{s}^{2} \kappa} \int d^{2} \theta\left(c_{s}+c_{s}^{\prime} \theta^{2}\right) \operatorname{Tr}\left(W^{\alpha} W_{\alpha}\right)_{s}\left(H_{u} \cdot H_{d}\right)+\text { h.c. } \\
& =\sum_{s=1,2} \frac{c_{s}}{4 M^{2}}\left\{\left(h_{u} \cdot h_{d}\right)\left[i\left(\lambda_{s}^{a} \sigma^{\mu} \mathcal{D}_{\mu} \bar{\lambda}_{s}^{a}-\mathcal{D}_{\mu} \bar{\lambda}_{s}^{a} \bar{\sigma}^{\mu} \lambda_{s}^{a}\right)+D_{s}^{a} D_{s}^{a}-\frac{1}{2}\left(F_{s}^{a \mu \nu} F_{s \mu \nu}^{a}+\frac{i \epsilon^{\mu \nu \rho \sigma}}{2} F_{s \mu \nu}^{a} F_{s \rho \sigma}^{a}\right)\right]\right. \\
& \left.-\sqrt{2}\left(h_{u} \cdot \psi_{d}+\psi_{u} \cdot h_{d}\right)\left(\lambda_{s}^{a} D_{s}^{a}+\sigma^{\mu \nu} \lambda_{s}^{a} F_{s \mu \nu}^{a}\right)+\left(h_{u} \cdot F_{d}+F_{u} \cdot h_{d}-\psi_{u} \cdot \psi_{d}\right) \lambda_{s}^{a} \lambda_{s}^{a}\right\} \\
& +\frac{c_{s}^{\prime}}{4 M^{2}}\left(h_{u} \cdot h_{d}\right)\left(\lambda_{s}^{a} \lambda_{s}^{a}\right)+\text { h.c. }
\end{aligned}
$$

Above we introduced $\mathcal{D}_{\mu} \bar{\lambda}^{a}=\partial_{\mu} \bar{\lambda}^{a}-g t^{a b c} V_{\mu}^{b} \bar{\lambda}^{c}$ for covariant derivatives of the gauginos.

From $\mathcal{L}$ one finds the equations of motion for the auxiliary fields of Higgs superfields:

$$
\begin{aligned}
F_{d}^{* q} & =-\epsilon^{q p} h_{u}^{p}\left[\mu+2 \frac{c_{0}}{M}\left(h_{d} \cdot h_{u}\right)-\frac{c_{2}}{4 M^{2}} \lambda_{2}^{a} \lambda_{2}^{a}-\frac{c_{1}}{4 M^{2}} \lambda_{1}^{2}\right] \\
F_{u}^{* q} & =-\epsilon^{p q} h_{d}^{p}\left[\mu+2 \frac{c_{0}}{M}\left(h_{d} \cdot h_{u}\right)-\frac{c_{2}}{4 M^{2}} \lambda_{2}^{a} \lambda_{2}^{a}-\frac{c_{1}}{4 M^{2}} \lambda_{1}^{2}\right]
\end{aligned}
$$

where $q$ is a $\mathrm{SU}(2)_{L}$ doublet index. For the auxiliary fields of the vector superfields we find: 


$$
\begin{gathered}
D_{2}^{a}=-\left[g_{2}\left(h_{d}^{\dagger} T^{a} h_{d}+h_{u}^{\dagger} T^{a} h_{u}\right)\left(1-\frac{c_{2}}{2 M^{2}}\left(h_{u} \cdot h_{d}+\text { h.c. }\right)\right)\right. \\
\left.-\frac{\sqrt{2} c_{2}}{4 M^{2}}\left(\left(h_{u} \cdot \psi_{d}+\psi_{u} \cdot h_{d}\right) \lambda_{2}^{a}+\text { h.c. }\right)\right] \\
D_{1}=-\left[g_{1}\left(h_{d}^{\dagger}\left(-\frac{1}{2}\right) h_{d}+h_{u}^{\dagger}\left(\frac{1}{2}\right) h_{u}\right)\left(1-\frac{c_{1}}{2 M^{2}}\left(h_{u} \cdot h_{d}+\text { h.c. }\right)\right)\right. \\
\left.\left.-\frac{\sqrt{2} c_{1}}{4 M^{2}}\left(h_{u} \cdot \psi_{d}+\psi_{u} \cdot h_{d}\right) \lambda_{1}^{a}+\text { h.c. }\right)\right]
\end{gathered}
$$

with $T^{a}=\sigma^{a} / 2$. The squares become

$$
\begin{aligned}
D_{2}^{a} D_{2}^{a} & =\frac{g_{2}^{2}}{4}\left[1-\left(\frac{c_{2}}{2 M^{2}} h_{u} \cdot h_{d}+\text { h.c. }\right)\right]^{2}\left[\left(\left|h_{d}\right|^{2}-\left|h_{u}\right|^{2}\right)^{2}+4\left|h_{d}^{\dagger} h_{u}\right|^{2}\right] \\
& -\frac{\sqrt{2}}{2} g_{2}\left[h_{d}^{\dagger} T^{a} h_{d}+h_{u}^{\dagger} T^{a} h_{u}\right]\left[\frac{c_{2}}{2 M^{2}}\left(h_{u} \cdot \psi_{d}+\psi_{u} \cdot h_{d}\right) \lambda_{2}^{a}+\text { h.c. }\right] \\
D_{1}^{2}= & \frac{g_{1}^{2}}{4}\left[1-\left(\frac{c_{1}}{2 M^{2}} h_{u} \cdot h_{d}+\text { h.c. }\right)\right]^{2}\left(\left|h_{d}\right|^{2}-\left|h_{u}\right|^{2}\right)^{2} \\
& -\frac{\sqrt{2}}{2} g_{1}\left[h_{d}^{\dagger}\left(-\frac{1}{2}\right) h_{d}+h_{u}^{\dagger}\left(\frac{1}{2}\right) h_{u}\right]\left[\frac{c_{1}}{2 M^{2}}\left(h_{u} \cdot \psi_{d}+\psi_{u} \cdot h_{d}\right) \lambda_{1}+\text { h.c. }\right]
\end{aligned}
$$

$\mathcal{O}_{5}$ and $\mathcal{O}_{6}$ and eqs. A.4 to A.8 give the corrections to the MSSM Higgs Lagrangian. Using the corrected auxiliary fields in the usual MSSM Higgs Lagrangian, additional terms suppressed by $1 / M$ and $1 / M^{2}$ are generated. The full on-shell Lagrangian is then:

$$
\mathcal{L}=\mathcal{L}_{D}+\mathcal{L}_{F}+\mathcal{L}_{1}+\mathcal{L}_{2}+\mathcal{L}_{3}+\mathcal{L}_{\mathrm{SSB}}
$$

Eliminating the D-dependent terms in $\mathcal{L}$ one finds, see eqs. A.5 to A.8:

$$
\mathcal{L}_{D}=\sum_{s=1,2}-\frac{1}{2} D_{s}^{a} D_{s}^{a}\left[1+\frac{c_{s}}{2 M^{2}}\left(h_{u} \cdot h_{d}+\text { h.c. }\right)\right]
$$

and use A.8. Eliminating the $F$-dependent terms in $\mathcal{L}$ gives $\mathcal{L}_{F}$ :

$$
\begin{aligned}
-\mathcal{L}_{F} & \equiv\left|F_{d}\right|^{2}+\left|F_{u}\right|^{2}=\left|\mu+2 \frac{c_{0}}{M} h_{d} \cdot h_{u}\right|^{2}\left(\left|h_{d}\right|^{2}+\left|h_{u}\right|^{2}\right) \\
& +\left[\mu\left(-\frac{c_{2}}{4 M^{2}} \lambda_{2}^{a} \lambda_{2}^{a}-\frac{c_{1}}{4 M^{2}} \lambda_{1}^{2}\right)\left(\left|h_{d}\right|^{2}+\left|h_{u}\right|^{2}\right)+\text { h.c. }\right]
\end{aligned}
$$

Apart from auxiliary field contributions, there are also terms in the Lagrangian with spacetime derivatives, that contribute to the kinetic terms for Weyl fermions $\psi_{u, d}, \lambda_{1,2}^{a}$ when the neutral singlet $h_{u, d}^{0}$ components of $h_{u, d}$ acquire a vev:

$$
\mathcal{L}_{1}=\frac{c_{2}}{4 M^{2}}\left(h_{u} \cdot h_{d}\right)\left[i\left(\lambda_{2}^{a} \sigma^{\mu} \mathcal{D}_{\mu} \bar{\lambda}_{2}^{a}-\mathcal{D}_{\mu} \bar{\lambda}_{2}^{a} \bar{\sigma}^{\mu} \lambda_{2}^{a}\right)\right]+\text { h.c. }+(2 \rightarrow 1)
$$


When the Higgs neutral singlets acquire a vev, these terms produce wavefunction renormalization of Weyl kinetic terms and a threshold correction to gauge couplings $g_{1}$ and $g_{2}$.

There are also terms contributing to fermion masses when the Higgs fields acquire vev's

$$
\begin{aligned}
\mathcal{L}_{2} & =\frac{c_{2}^{\prime}}{4 M^{2}}\left(h_{u} \cdot h_{d}\right)\left(\lambda_{2}^{a} \lambda_{2}^{a}\right)+\frac{c_{1}^{\prime}}{4 M^{2}}\left(h_{u} \cdot h_{d}\right)\left(\lambda_{1} \lambda_{1}\right) \\
& +\frac{c_{0}}{M}\left[2\left(h_{u} \cdot h_{d}\right)\left(-\psi_{u} \cdot \psi_{d}\right)-\left(h_{u} \cdot \psi_{d}+\psi_{u} \cdot h_{d}\right)^{2}\right]+\text { h.c. }
\end{aligned}
$$

Further, there are some interaction terms generated

$$
\begin{aligned}
\mathcal{L}_{3} & =\left\{\frac { c _ { 2 } } { 4 M ^ { 2 } } \left[-\frac{1}{2}\left(h_{u} \cdot h_{d}\right)\left(F_{2}^{a \mu \nu} F_{2 \mu \nu}^{a}+\frac{i}{2} \epsilon^{\mu \nu \rho \sigma} F_{2 \mu \nu}^{a} F_{2 \rho \sigma}^{a}\right)\right.\right. \\
& \left.\left.-\sqrt{2}\left(h_{u} \cdot \psi_{d}+\psi_{u} \cdot h_{d}\right) \sigma^{\mu \nu} \lambda_{2}^{a} F_{2 \mu \nu}^{a}-\psi_{u} \cdot \psi_{d} \lambda_{2}^{a} \lambda_{2}^{a}\right]+(2 \rightarrow 1)+\text { h.c. }\right\}
\end{aligned}
$$

Finally, the Lagrangian contains ( $F$ and $D$ independent) corrections from supersymmetry breaking due to spurion dependence in the dimension 5 operator as well as the usual soft terms of the MSSM. All these together give $\mathcal{L}_{\mathrm{SSB}}$ :

$$
\mathcal{L}_{\mathrm{SSB}}=-V_{\mathrm{SSB}}=\left[\frac{c_{0}^{\prime}}{M}\left(h_{u} \cdot h_{d}\right)^{2}+\mu B\left(h_{d} \cdot h_{u}\right)+\text { h.c. }\right]-m_{d}^{2}\left|h_{d}\right|^{2}-m_{u}^{2}\left|h_{u}\right|^{2}
$$

This concludes the presentation of the Lagrangian to $1 / M^{2}$ order. From $\mathcal{L}$ we find the scalar potential $V_{h}$ for the Higgs sector shown in the text, eq. (2.6) in which as usual $h_{u, d}$ denote $\mathrm{SU}(2)$ doublets. From this one obtains:

$$
m_{h}^{2}=m_{h, \text { loop }}^{2}+\Delta m_{h}^{2}
$$

with:

$$
\begin{aligned}
m_{h, \text { loop }}^{2} & =\frac{1}{2}\left\{m_{A}^{2}+m_{Z}^{2}+\delta m_{Z}^{2} \sin ^{2} \beta-\sqrt{w}\right\} \\
w & \equiv\left[\left(m_{A}^{2}-m_{Z}^{2}\right) \cos 2 \beta+\delta m_{Z}^{2} \sin ^{2} \beta\right]^{2}+\sin ^{2} 2 \beta\left(m_{A}^{2}+m_{Z}^{2}\right)^{2}
\end{aligned}
$$

and

$$
\begin{aligned}
\Delta m_{h}^{2} & =f_{1}\left(2 \mu \frac{c_{0}}{M}\right)+f_{2}\left(-2 \frac{c_{0}^{\prime}}{M}\right)+f_{3}\left(2 \mu \frac{c_{0}}{M}\right)^{2}+f_{4}\left(-2 \frac{c_{0}^{\prime}}{M}\right)^{2}+f_{5}\left(2 \mu \frac{c_{0}}{M}\right)\left(-2 \frac{c_{0}^{\prime}}{M}\right) \\
& +f_{6}\left(g_{1}^{2} \frac{c_{1}}{M^{2}}+g_{2}^{2} \frac{c_{2}}{M^{2}}\right)+\mathcal{O}\left(\frac{1}{M^{3}}\right)
\end{aligned}
$$

where 


$$
\begin{aligned}
& f_{1}=v^{2} \sin 2 \beta\left\{1+\frac{\left(m_{A}^{2}+m_{Z}^{2}\right)}{\sqrt{w}}\right\} \\
& f_{2}=\frac{v^{2}}{2}\left\{1-\frac{\cos 2 \beta}{\sqrt{w}}\left[\left(m_{A}^{2}-m_{Z}^{2}\right) \cos 2 \beta+m_{Z}^{2} \delta \sin ^{2} \beta\right]\right\} \\
& f_{3}=\frac{v^{4}}{4 \mu^{2}} \sin ^{2} 2 \beta+\frac{v^{4}}{\sqrt{w}}\left\{-1+\frac{1}{2 \mu^{2}}\left(m_{A}^{2}+m_{Z}^{2}\right) \sin ^{2} 2 \beta\right\}+\frac{1}{w^{3 / 2}}\left(m_{A}^{2}+m_{Z}^{2}\right)^{2} v^{4} \sin ^{2} 2 \beta \\
& f_{4}=-\frac{v^{4}}{16 w^{3 / 2}}\left(m_{A}^{2}+m_{Z}^{2}\right)^{2} \sin ^{2} 4 \beta \\
& f_{5}=-\frac{v^{4}}{4 w^{3 / 2}}\left(m_{A}^{2}+m_{Z}^{2}\right)\left[\delta m_{Z}^{2}+\left(2 m_{A}^{2}-(2+\delta) m_{Z}^{2}\right) \cos 2 \beta\right] \sin 4 \beta \\
& f_{6}=\frac{v^{4}}{32} \sin 2 \beta\left[1+\frac{1}{4 \sqrt{w}}\left[8 m_{A}^{2}-(4+3 \delta) m_{Z}^{2}+6 \delta m_{Z}^{2} \cos 2 \beta+3\left(4 m_{A}^{2}-\delta m_{Z}^{2}\right) \cos 4 \beta\right]\right] \text { (A.18) }
\end{aligned}
$$

and finally

$$
m_{A}^{2}=\frac{2 B \mu}{\sin 2 \beta}-\frac{v^{2}}{\sin 2 \beta}\left(2 \mu \frac{c_{0}}{M}\right)+\left(2 \frac{c_{0}^{\prime}}{M}\right) v^{2}-\frac{v^{4}}{32} \frac{\cos ^{2} 2 \beta}{\sin 2 \beta}\left(\frac{g_{1}^{2} c_{1}}{M^{2}}+\frac{c_{2} g_{2}^{2}}{M^{2}}\right)+\mathcal{O}\left(\frac{1}{M^{3}}\right)
$$

\section{A.2 1-loop form factors}

The form factors in 3.4 are given by,

$$
\begin{aligned}
\mathcal{A}_{g}^{(t)}=\frac{3}{4} \mathcal{A}_{1 / 2}\left(\tau_{t}\right) & , \quad \mathcal{A}_{g}^{(b)}=\frac{3}{4} \mathcal{A}_{1 / 2}\left(\tau_{b}\right) \\
\mathcal{A}_{\gamma}^{(W)}=\mathcal{A}_{1}\left(\tau_{W}\right) & , \quad \mathcal{A}_{\gamma}^{(t)}=N_{c} Q_{t}^{2} \mathcal{A}_{1 / 2}\left(\tau_{t}\right) \\
\mathcal{A}_{Z \gamma}^{(W)}=\cos \theta_{w} A_{1}\left(\tau_{W}, \lambda_{W}\right) & , \quad \mathcal{A}_{Z \gamma}^{(t)}=N_{c} \frac{Q_{t}\left(2 T_{3}^{(t)}-4 Q_{t} \sin ^{2} \theta_{w}\right)}{\cos \theta_{w}} A_{1 / 2}\left(\tau_{t}, \lambda_{t}\right)
\end{aligned}
$$

where $\tau_{i}=4 m_{i}^{2} / m_{h}^{2}, \lambda_{i}=4 m_{i}^{2} / m_{Z}^{2}, N_{c}=3, Q_{t}=2 / 3, T_{3}^{(t)}=1 / 2$ and

$$
\begin{aligned}
\mathcal{A}_{1 / 2}(\tau) & =2 \tau^{2}\left[\tau^{-1}+\left(\tau^{-1}-1\right) f\left(\tau^{-1}\right)\right] \\
\mathcal{A}_{1}(\tau) & =-\tau^{2}\left[2 \tau^{-2}+3 \tau^{-1}+3\left(2 \tau^{-1}-1\right) f\left(\tau^{-1}\right)\right] \\
\mathcal{A}_{1 / 2}(\tau, \lambda) & =I_{1}(\tau, \lambda)-I_{2}(\tau, \lambda) \\
\mathcal{A}_{1}(\tau, \lambda) & =4\left(3-\tan ^{2} \theta_{w}\right) I_{2}(\tau, \lambda)+\left[\left(1+2 \tau^{-1}\right) \tan ^{2} \theta_{w}-\left(5+2 \tau^{-1}\right)\right] I_{1}(\tau, \lambda)(\mathrm{A} .20)
\end{aligned}
$$

where

$$
\begin{aligned}
I_{1}(\tau, \lambda) & =\frac{\tau \lambda}{2(\tau-\lambda)}+\frac{\tau^{2} \lambda^{2}}{2(\tau-\lambda)^{2}}\left[f\left(\tau^{-1}\right)-f\left(\lambda^{-1}\right)\right]+\frac{\tau^{2} \lambda}{(\tau-\lambda)^{2}}\left[g\left(\tau^{-1}\right)-g\left(\lambda^{-1}\right)\right] \\
I_{2}(\tau, \lambda) & =-\frac{\tau \lambda}{2(\tau-\lambda)}\left[f\left(\tau^{-1}\right)-f\left(\lambda^{-1}\right)\right]
\end{aligned}
$$


and

$$
\begin{aligned}
& f(x)= \begin{cases}\arcsin ^{2} \sqrt{x} & x \leq 1 \\
-\frac{1}{4}\left[\log \frac{1+\sqrt{1-x^{-1}}}{1-\sqrt{1-x^{-1}}}-i \pi\right]^{2} & x>1,\end{cases} \\
& g(x)= \begin{cases}\sqrt{x^{-1}-1} \arcsin \sqrt{x} & x \leq 1 \\
\frac{\sqrt{1-x^{-1}}}{2}\left[\log \frac{1+\sqrt{1-x^{-1}}}{1-\sqrt{1-x^{-1}}}-i \pi\right]^{2} & x>1 .\end{cases}
\end{aligned}
$$

\section{References}

[1] G. Aad et al. [ATLAS Collaboration], Phys. Lett. B 716 (2012) 1 arXiv:1207.7214 [hep-ex]]. S. Chatrchyan et al. [CMS Collaboration], Phys. Lett. B 716 (2012) 30 arXiv:1207.7235 [hep-ex]].

[2] J. Baglio, A. Djouadi and R. M. Godbole, Phys. Lett. B 716 (2012) 203 arXiv:1207.1451 [hep-ph]].

[3] U. Ellwanger, JHEP 1203 (2012) 044 arXiv:1112.3548 [hep-ph]]. K. Blum and R. T. D'Agnolo, Phys. Lett. B 714 (2012) 66 [arXiv:1202.2364 [hep-ph]]. J. -J. Cao, Z. -X. Heng, J. M. Yang, Y. -M. Zhang and J. -Y. Zhu, JHEP 1203 (2012) 086 [arXiv:1202.5821 [hep-ph]]. F. Boudjema and G. D. La Rochelle, Phys. Rev. D 86 (2012) 015018 arXiv:1203.3141 [hep-ph]]. B. Bellazzini, C. Csaki, J. Hubisz, J. Serra and J. Terning, JHEP 1211 (2012) 003 [arXiv:1205.4032 [hep-ph]]. A. Azatov, S. Chang, N. Craig and J. Galloway, Phys. Rev. D 86 (2012) 075033 arXiv:1206.1058 [hep-ph]]. K. Hagiwara, J. S. Lee and J. Nakamura, JHEP 1210 (2012) 002 arXiv:1207.0802 [hepph]]. R. Benbrik, M. Gomez Bock, S. Heinemeyer, O. Stal, G. Weiglein and L. Zeune, arXiv:1207.1096 [hep-ph]. M. R. Buckley and D. Hooper, Phys. Rev. D 86 (2012) 075008 arXiv:1207.1445 [hep-ph]]. H. An, T. Liu and L. -T. Wang, Phys. Rev. D 86 (2012) 075030 [arXiv:1207.2473 [hep-ph]]. A. Alves, A. G. Dias, E. R. Barreto, C. A. de S.Pires, F. S. Queiroz and P. S. R. da Silva, arXiv:1207.3699 [hep-ph]. T. Abe, N. Chen and H. J. He, arXiv:1207.4103 [hep-ph]. D. Bertolini and M. McCullough, arXiv:1207.4209 [hepph]. A. Joglekar, P. Schwaller and C. E. M. Wagner, arXiv:1207.4235 [hep-ph]. N. ArkaniHamed, K. Blum, R. T. D'Agnolo and J. Fan, arXiv:1207.4482 [hep-ph]. N. Craig and S. Thomas, arXiv:1207.4835 [hep-ph]. B. Batell, D. McKeen and M. Pospelov, JHEP 1210 (2012) 104 arXiv:1207.6252 [hep-ph]]. B. Bellazzini, C. Petersson and R. Torre, Phys. Rev. D 86 (2012) 033016 [arXiv:1207.0803 [hep-ph]]. M. Hashimoto and V. A. Miransky, arXiv:1208.1305 [hep-ph]. M. Reece, arXiv:1208.1765 [hep-ph]. F. Boudjema and G. D. La Rochelle, arXiv:1208.1952 [hep-ph]. A. Alves, arXiv:1209.1037 [hep-ph]. E. Bertuzzo, P. A. N. Machado and R. Zukanovich Funchal, arXiv:1209.6359 
[hep-ph]. K. Schmidt-Hoberg and F. Staub, JHEP 1210 (2012) 195 arXiv:1208.1683 [hep-ph]]. P. Bechtle, S. Heinemeyer, O. Stål, T. Stefaniak, G. Weiglein and L. Zeune, arXiv:1211.1955 [hep-ph]. C. Petersson, A. Romagnoni and R. Torre, arXiv:1211.2114 [hep-ph]. K. Schmidt-Hoberg, F. Staub and M. W. Winkler, arXiv:1211.2835 [hep-ph]. E. Dudas, C. Petersson and P. Tziveloglou, arXiv:1211.5609 [hep-ph].

[4] J. F. Gunion and H. E. Haber, Phys. Rev. D 67 (2003) 075019 hep-ph/0207010.

[5] M. Carena, S. Gori, N. R. Shah and C. E. M. Wagner, JHEP 1203 (2012) 014 arXiv:1112.3336 [hep-ph]]. M. Carena, S. Gori, N. R. Shah, C. E. M. Wagner and L. -T. Wang, JHEP 1207 (2012) 175 [arXiv:1205.5842 [hep-ph]]. M. Carena, S. Gori, I. Low, N. R. Shah and C. E. M. Wagner, arXiv:1211.6136 [hep-ph].

[6] For a two-loop analysis see: D. M. Ghilencea, H. M. Lee and M. Park, JHEP 1207 (2012) 046 [arXiv:1203.0569 [hep-ph]]. S. Cassel, D. M. Ghilencea and G. G. Ross, Phys. Lett. B 687 (2010) 214, arXiv:0911.1134 [hep-ph]] and Nucl. Phys. B 835 (2010) 110, arXiv:1001.3884 [hep-ph]]. S. Cassel and D. M. Ghilencea, Mod. Phys. Lett. A 27 (2012) 1230003 arXiv:1103.4793 [hep-ph]].

[7] L. J. Hall, D. Pinner and J. T. Ruderman, JHEP 1204 (2012) 131 arXiv:1112.2703 [hep-ph]].

[8] D. M. Ghilencea and G. G. Ross, Nucl. Phys. B 868 (2013) 65 arXiv:1208.0837 [hep-ph]].

[9] U. Ellwanger, C. Hugonie and A. M. Teixeira, Phys. Rept. 496 (2010) 1 arXiv:0910.1785 [hep-ph]].

[10] I. Antoniadis, E. Dudas, D. M. Ghilencea and P. Tziveloglou, Nucl. Phys. B 841 (2010) 157 arXiv:1006.1662 [hep-ph]]. C. Petersson and A. Romagnoni, JHEP 1202 (2012) 142 arXiv:1111.3368 [hep-ph]]. C. Petersson, A. Romagnoni and R. Torre, JHEP 1210 (2012) 016 arXiv:1203.4563 [hep-ph]].

[11] See for example S. F. King, M. Muhlleitner, R. Nevzorov and K. Walz, arXiv:1211.5074 [hep-ph].

[12] G. G. Ross and K. Schmidt-Hoberg, Nucl. Phys. B 862 (2012) 710 arXiv:1108.1284 [hep-ph]]. G. G. Ross, K. Schmidt-Hoberg and F. Staub, JHEP 1208 (2012) 074 arXiv:1205.1509 [hep-ph]].

[13] S. Cassel, D. M. Ghilencea, G. G. Ross, Nucl. Phys. B 825 (2010) 203 arXiv:0903.1115 [hep-ph]].

[14] A. Delgado, C. Kolda, J. P. Olson, A. de la Puente, Phys. Rev. Lett. 105 (2010) 091802. arXiv:1005.1282 [hep-ph]] 
[15] D. Piriz and J. Wudka, Phys. Rev. D 56 (1997) 4170 [arXiv:hep-ph/9707314.

[16] A. Brignole, J. A. Casas, J. R. Espinosa and I. Navarro, Nucl. Phys. B 666 (2003) 105 arXiv:hep-ph/0301121.

[17] M. Dine, N. Seiberg and S. Thomas, Phys. Rev. D 76 (2007) 095004 arXiv:0707.0005 [hep-ph]].

[18] M. Carena, K. Kong, E. Ponton, J. Zurita, Phys. Rev. D81 (2010) 015001. arXiv:0909.5434 [hep-ph]]. I. Antoniadis, E. Dudas, D. M. Ghilencea, P. Tziveloglou, Nucl. Phys. B831 (2010) 133-161. arXiv:0910.1100 [hep-ph]] and Nucl. Phys. B848 (2011) 1-32. arXiv:1012.5310 [hep-ph]]. M. Carena, E. Ponton and J. Zurita, Phys. Rev. D 85 (2012) 035007 arXiv:1111.2049 [hep-ph]]. I. Antoniadis, E. Dudas, D. M. Ghilencea, JHEP 0803 (2008) 045. arXiv:0708.0383 [hep-th]]. M. Berg, J. Edsjo, P. Gondolo, E. Lundstrom and S. Sjors, arXiv:0906.0583 [hep-ph].

[19] I. Antoniadis, E. Dudas, D. M. Ghilencea and P. Tziveloglou, Nucl. Phys. B 831 (2010) 133 arXiv:0910.1100 [hep-ph]]. J. J. Heckman, P. Kumar and B. Wecht, JHEP 1207 (2012) 118 arXiv:1204.3640 [hep-ph]].

[20] I. Antoniadis, E. Dudas, D. M. Ghilencea and P. Tziveloglou, Nucl. Phys. B 808 (2009) 155 arXiv:0806.3778 [hep-ph]].

[21] Y. Okada, M. Yamaguchi, T. Yanagida, Prog. Theor. Phys. 85 (1991) 1-6 and Phys. Lett. B262 (1991) 54-58. J. R. Ellis, G. Ridolfi, F. Zwirner, Phys. Lett. B257 (1991) 83-91 and Phys. Lett. B262 (1991) 477-484. H. E. Haber, R. Hempfling, Phys. Rev. Lett. 66 (1991) 1815-1818. M. S. Carena, J. R. Espinosa, M. Quiros and C. E. M. Wagner, Phys. Lett. B 355 (1995) 209 arXiv:hep-ph/9504316]. G. F. Giudice and R. Rattazzi, Nucl. Phys. B 757 (2006) 19 arXiv:hep-ph/0606105].

[22] I. Low, J. Lykken and G. Shaughnessy, arXiv:1207.1093 [hep-ph]. P. P. Giardino, K. Kannike, M. Raidal and A. Strumia, arXiv:1207.1347 [hep-ph]. M. Montull and F. Riva, JHEP 1211 (2012) 018 arXiv:1207.1716 [hep-ph]]. J. R. Espinosa, C. Grojean, M. Muhlleitner and M. Trott, arXiv:1207.1717 [hep-ph]. D. Carmi, A. Falkowski, E. Kuflik, T. Volansky and J. Zupan, JHEP 1210 (2012) 196 arXiv:1207.1718 [hep-ph]]. LHC Higgs Cross Section Working Group, A. David, A. Denner, M. Duehrssen, M. Grazzini, C. Grojean, G. Passarino and M. Schumacher et al., arXiv:1209.0040 [hep-ph]. G. Cacciapaglia, A. Deandrea, G. D. La Rochelle and J. -B. Flament, arXiv:1210.8120 [hep-ph]. T. Corbett, O. J. P. Eboli, J. Gonzalez-Fraile and M. C. Gonzalez-Garcia, arXiv:1211.4580 [hep-ph].

[23] A. Djouadi, Phys. Rept. 457 (2008) 1 hep-ph/0503172. M. Carena, I. Low and C. E. M. Wagner, JHEP 1208 (2012) 060 arXiv:1206.1082 [hep-ph]]. 
[24] R. N. Cahn, M. S. Chanowitz and N. Fleishon, Phys. Lett. B 82 (1979) 113. L. Bergstrom and G. Hulth, Nucl. Phys. B 259 (1985) 137 [Erratum-ibid. B 276 (1986) 744]. J. S. Gainer, W. -Y. Keung, I. Low and P. Schwaller, Phys. Rev. D 86 (2012) 033010 arXiv:1112.1405 [hep-ph]].

[25] M. Frank, T. Hahn, S. Heinemeyer, W. Hollik, H. Rzehak and G. Weiglein, JHEP 0702 (2007) 047 hep-ph/0611326. G. Degrassi, S. Heinemeyer, W. Hollik, P. Slavich and G. Weiglein, Eur. Phys. J. C 28 (2003) 133 hep-ph/0212020]. S. Heinemeyer, W. Hollik and G. Weiglein, Eur. Phys. J. C 9 (1999) 343 hep-ph/9812472]. S. Heinemeyer, W. Hollik and G. Weiglein, Comput. Phys. Commun. 124 (2000) 76 [hep-ph/9812320]. 